\title{
Approximate Stochastic Response of Hysteretic System With Fractional Element and Subjected to Combined Stochastic and Periodic Excitation
}

Fan Kong ( $\sim$ kongfan@whut.edu.cn )

Wuhan University of Technology https://orcid.org/0000-0002-9036-7273

\section{Renjie Han}

Wuhan University of Technology

\section{Yuanjin Zhang}

Wuhan University of Technology

\section{Research Article}

Keywords: Bouc-Wen model, fractional derivative, statistical linearization, harmonic balance, combined excitation

Posted Date: February 10th, 2021

DOI: https://doi.org/10.21203/rs.3.rs-181868/v1

License: (9) This work is licensed under a Creative Commons Attribution 4.0 International License. Read Full License

Version of Record: A version of this preprint was published at Nonlinear Dynamics on November 10th, 2021. See the published version at https://doi.org/10.1007/s11071-021-07014-w. 


\title{
Approximate stochastic response of hysteretic system with fractional element and subjected to combined stochastic and periodic excitation
}

\author{
Fan Kong · Han Renjie - Zhang Yuanjin
}

Received: date / Accepted: date

\begin{abstract}
A method based on statistical linearization is proposed, for determining response of the single-degreeof-freedom (SDOF) hysteretic system endowed with fractional derivatives and subjected to combined periodic and white/colored excitation. The method is developed by decomposing the system response into a combination of a periodic and of a zero-mean stochastic components. In this regard, first, the equation of motion is cast into two sets of coupled fractional-order non-linear differential equations with unknown deterministic and stochastic response components. Next, the harmonic balance method and the statistical linearization for the fractional-order deterministic and stochastic subsystems are used, to obtain the Fourier coefficients of the deterministic component and the variance of the stochastic component, respectively. This yields two sets of coupled non-linear algebraic equations which can be solved by appropriate standared numerical method. Pertinent numerical examples, including both softening and hardening Bouc-Wen hysteretic system endowed with different
\end{abstract}

Fan Kong · Han Renjie

School of Civil Engineering and Architecture, Wuhan University of Technology, 122 Luoshi Road, Wuhan, Hubei 430070, China.

Kong Fan

E-mail: kongfan@whutedu.cn

Han Renjie

E-mail: Hanrenjie@whut.edu.cn

Yuanjin Zhang

School of Safety Science and Emergency Management, Wuhan University of Technology, 122 Luoshi Road, Wuhan 430070, China

E-mail: ylzhyj@whut.edu.cn fractional-orders, are used to demonstrate the applicability and accuracy of the proposed method.

Keywords Bouc-Wen model · fractional derivative . statistical linearization · harmonic balance · combined excitation

\section{Introduction}

Many mechanical and structural systems subjected to severe dynamic loads exhibit hysteretic behavior [1]. The hysteretic restoring force not only depends on the instantaneous system response, but also depends on the response history. Therefore, compared to the zero- memory non-linear elements with elastic behavior, the hysteretic force-displacement relationships exhibit multivalued loops [2]. To describe the history-depended nonlinear behavior of the hysteretic systems, various parametric models have been proposed. A dry friction based hysteretic bilinear model can be found in Ref. [3, 4]. Proposed by Bouc [5] and improved by Wen [6], the BoucWen hysteretic model and its extensions [7] has gained wide popularity among researchers, due to its versatility and simplicity; Also see a comprehensive literature review in [8] and a book 9] devoted to various application of this celebrated model.

A proper hysteretic model can represent various of engineering structural/material hysteresis behaviors. In this regard, the Bouc-Wen model belongs to a class of smooth analytical models described by differential equations 10]. This yields a mathematical based framework to develop analytical knowledge on relevant properties, which can be used for multi-purposes in engineering applications. In this regard, Chassiakos 11 et al. developed an on-line identification method for hysteretic systems using the Bouc-Wen model. Later on, 
the Bouc-Wen model was utilized for representing forcedisplacement relationship of MR dampers [12, leadcore rubber isolators [13] and steel dampers [14 and for describing constitutive law of smart materials [15].

Recently, a useful mathematical tools named fractionalorder calculus has been widely used in various engineering applications, especially for mechanical modeling of visco-elastic materials 16. In this context, one may argue that if visco-elastic relaxation test of the material is well fitted by a power law decay, then the fractional constitutive law in Caputo's derivative sense naturally appears [17. Compared to the standard linear solid model, the fractional derivative model can describe the broad-band of visco-elastic materials with small number of parameters [18. Further, fractional derivatives has proved particularly useful in the fields of structural engineering, where visco-elastic materials are used in vibration mitigation devices for structures. Certain examples are related to visco-elastic dampers for connecting shear walls [19] and adjacent stories 20] of buildings. In this regards, the response determination of a structural dynamic systems endowed with fractional derivatives and subjected to random excitation arise naturally; see 21] for example.

For the engineering applications where the viscoelastic controlled/isolated structures or/and the involved visco-elastic dampers/isolators behave hysteretically, one need to study a hysteretic dynamic model with fractional derivative. This kind of model has a rate-dependent force- displacement relationship, which can be used for modeling visco-elastic controlled/isolated structures subjected to server external excitation. Very recently, Kang 22. et al. presented a new fractional-order normalized Bouc-Wen model to describe the asymmetric and ratedependent hysteresis nonlinearity of piezoelectric actuators.

From the preceding literature review one may conclude that very few researchers focus on the response of a hysteretic dynamic system endowed with integer/fractional- damping element subject to purely periodic or stochastic excitation. However, for some engineering applications, structures/systems are subjected to combined periodic and stochastic excitation. For example, aircrafts with rotation mechanism 23] are often subjected to the mixture of colored stochastic and harmonic excitation. Other examples include response of a gear system [24, 25] and airfoil model [26] under stochastic excitation with uncertain disturbance, and so forth. More recently, non-linear systems subjected to combined harmonic and stochastic excitation attract increasing interest of researchers to account a more realistic operation condition in energy harvest application 27, 28]. The preceding literature review ne- cessitate the following research regarding the response determination of a hysteretic systems endowed with fractional element and subjected to combined periodic and stochastic excitation.

In this paper, a method based on statistical linearization(SL) 29] is proposed, for determining response of the single-degree-of-freedom (SDOF) hysteretic system endowed with fractional element and subjected to combined periodic and white/colored excitation. It can be regarded as an extension of the recent developed method for response determination of a integer-order hysteretic dynamic system subjected to combined harmonic and white noise excitation [30]. This is achieved based on an assumption that the system response can be decomposed into a summation of deterministic and stochastic component 31]. Accordingly, the original fractional order equation of motion can be cast into a sets of two coupled fractional-order differential equations in terms of the deterministic component, and variance of the stochastic component, respectively. The harmonic balance method and the SL method are then utilized for these sub-equations of motion, leading to a set of two non-linear algebraic equations. These non-linear algebraic equations can be solved simultaneously using standard numerical schemes, such as Newton's iteration method. The applicability and accuracy of the proposed method is demonstrated by comparing with pertinent Monte Carlo simulations.

\section{Mathematical formulation}

Consider a SDOF hysteretic system endowed with fractional element and subjected to a combined periodic and stochastic excitation

$m \ddot{x}(t)+c \mathrm{D}_{\mathrm{C}}^{q} x(t)+\alpha k x(t)+(1-\alpha) k z(t)=w(t)+f(t)$,

where $x(t)$ and $\ddot{x}(t)$ is the displacement and acceleration of the system, respectively; $m, c$ and $k$ is the coefficient for mass, damping and stiffness, respectively; $D_{\mathrm{C}}(\cdot)$ represents the fractional order in Caputo's sense

$\mathrm{D}_{\mathrm{C}}^{q}[x(t)]=\frac{1}{\Gamma(1-q)} \frac{\mathrm{d}}{\mathrm{d} t} \int_{0}^{t} \frac{\dot{x}(\tau)}{(t-\tau)^{q}} \mathrm{~d} \tau ;$

The superscript $q$ denotes the order of fractional derivative; $\alpha$ is usually referred to as the 'rigidity ratio'; $w(t)$ is a white/colored excitation with power spectrum density $S_{w}(\omega, t) ; f(t)$ is a deterministic periodic excitation, written in a form of Fourier series

$f(t)=\sum_{n=1}^{N}\left[A_{n} \cos \omega_{n} t+B_{n} \sin \omega_{n} t\right]$ 
with $A_{n}$ and $B_{n}$ being the Fourier coefficients of the $n$th harmonic term; $z(t)$ is the hysteretic displacement governed in a differential form

$\dot{z}=g_{z}(z, \dot{x})$.

As an archetypal hysteretic model, the Bouc-Wen model has been widely used in structural engineering applications. In this context, consider

$\dot{z}(t)=\dot{x}\left[A-|z|^{n}(\gamma \operatorname{sgn}(\dot{x}) \operatorname{sgn}(z)+\beta)\right]$,

where $A, n, \gamma$ and $\beta$ are the Bouc-Wen hysteretic parameters. In particular, when $n=1$ Eq. (5) becomes

$$
\dot{z}(t)=A \dot{x}-\gamma z|\dot{x}|-\beta \dot{x}|z| .
$$

Note the damping coefficient $c=2 \zeta m \omega_{n}^{2-q}$ to ensure a consistent dimension of the fractional-order damping coefficient with the one of the integer-order derivative. $\omega_{n}=\sqrt{k / m}$ is the natural frequency and $\zeta$ is the damping ratio.

Assume next the steady-state response of the system, governed by Eq. (1), can be cast into a combination of a deterministic and of a stochastic component. That is

$$
\begin{aligned}
& x(t)=\hat{x}(t)+\mu_{x}(t), \\
& z(t)=\hat{z}(t)+\mu_{z}(t),
\end{aligned}
$$

where $\hat{x}(t)$ and $\hat{z}(t)$ are the zero-mean stochastic processes; $\mu_{x}(t)$ and $\mu_{z}(t)$ are the deterministic mean processes which can be further written as a Fourier series

$$
\begin{aligned}
& \mu_{x}(t)=\sum_{n=1}^{N}\left(C_{n} \cos \omega_{n} t+D_{n} \sin \omega_{n} t\right), \\
& \mu_{z}(t)=\sum_{n=1}^{N}\left(U_{n} \cos \omega_{n} t+V_{n} \sin \omega_{n} t\right),
\end{aligned}
$$

where $C_{n}, D_{n}, V_{n}, D_{n}$ are the Fourier coefficients of response. Substituting Eq.(7) into the equation of motion shown in Eq. (1) yields

$$
\begin{array}{r}
m\left(\mu_{\ddot{x}}+\ddot{\hat{x}}\right)+c \mathrm{D}_{\mathrm{L}}^{q}\left(\mu_{x}+\hat{x}\right)+\alpha k\left(\mu_{x}+\hat{x}\right)+ \\
(1-\alpha) k\left(\mu_{z}+\hat{z}\right)=w(t)+f(t) .
\end{array}
$$

Taking mathematical expection on both sides of Eq. (9) leads to

$m \mu_{\ddot{x}}(t)+c \mathrm{D}_{\mathrm{C}}^{q} \mu_{x}(t)+\alpha k \mu_{x}(t)+(1-\alpha) k \mu_{z}(t)=f(t)$.

Substracting Eq. 10] from Eq.90 yields

$m \ddot{\hat{x}}(t)+c \mathrm{D}_{\mathrm{C}}^{q} \hat{x}(t)+\alpha k \hat{x}(t)+(1-\alpha) k \hat{z}(t)=w(t)$.
Similary, substituting Eq.(7) into the auxiliary firstorder differential equation governing the hysteretic term shown in Eq. (6), yields

$$
\begin{array}{r}
\dot{\hat{z}}+\mu_{\dot{z}}=A\left(\dot{\hat{x}}+\mu_{\dot{x}}\right)- \\
\gamma\left(\mu_{z}+\hat{z}\right)\left|\dot{\hat{x}}+\mu_{\dot{x}}\right|- \\
\beta\left(\dot{\hat{x}}+\mu_{\dot{x}}\right)\left|\mu_{z}+\hat{z}\right| .
\end{array}
$$

Taking mathematical expectation on both sides of Eq. 12 leads to

$\mu_{\dot{z}}=A \mu_{\dot{x}}-\gamma \mathrm{E}[z|\dot{x}|]-\beta \mathrm{E}[\dot{x}|z|]$.

Substituting Eq. 13 form Eq. 12 yields

$\dot{\hat{z}}(t)=A \dot{\hat{x}}-\gamma z|\dot{x}|-\beta \dot{x}|z|+(\gamma \mathrm{E}[z|\dot{x}|]+\beta \mathrm{E}[\dot{x}|z|])$.

Note that these two sets of differential equations (Eqs. (10)-(11) and Eqs. 130-(14) ) are coupled, because the expectations of the non-linear functions include both $\mu_{x}, \mu_{z}$ and $\sigma_{\dot{\hat{x}}}, \sigma_{\hat{z}}$. Therefore, a simultaneous solution of them should be adopted. In the following part, the harmonic balance method is utilized for solving the Fourier coefficients of the deterministic component, while the statistical linearization method is used for the variance/covariance of the stochastic component.

\section{Harmonic balance method for the deterministic component}

An assumption often used in the statistical linearization is the Gaussian distribution of response. In this regard, the expectation in Eqs. (13) and (14) can be evaluated in an approximate closed-form [30. That is

$$
\begin{aligned}
& E[z|\dot{x}|]=\sqrt{\frac{2}{\pi}}\left(\rho \mu_{\dot{x}} \sigma_{\hat{z}}+\mu_{z} \sigma_{\dot{\hat{x}}}+\frac{\mu_{z} \mu_{\dot{x}}^{2}}{2 \sigma_{\dot{\hat{x}}}}\right), \\
& E[\dot{x}|z|]=\sqrt{\frac{2}{\pi}}\left(\rho \mu_{z} \sigma_{\dot{\hat{x}}}+\mu_{\dot{x}} \sigma_{\hat{z}}+\frac{\mu_{z} \mu_{\dot{x}}^{2}}{2 \sigma_{z}}\right),
\end{aligned}
$$

where $\sigma_{\dot{\hat{x}}}$ and $\sigma_{\hat{z}}$ are standard deviation of $\dot{\hat{x}}$ and $\hat{z}$, respectively; $\rho$ is the correlation coefficient of $\dot{x}$ and $z$.

Taking fractional-, first- and second- order derivative on both sides of Eq. 8a yields

$$
D_{C}^{q} \mu_{x}(t)=\sum_{n=1}^{N}\left[C_{n} \omega_{n}^{q} \cos \left(\omega_{n} t+\frac{\pi q}{2}\right)+D_{n} \omega_{n}^{q} \sin \left(\omega_{n} t+\frac{\pi q}{2}\right)\right]
$$

$$
\mu_{\dot{x}}(t)=\sum_{n=1}^{N}\left[-C_{n} \omega_{n} \sin \omega_{n} t+D_{n} \omega_{n} \cos \omega_{n} t\right]
$$


and

$$
\mu_{\ddot{x}}(t)=\sum_{n=1}^{N}\left[-C_{n} \omega_{n}^{2} \cos \omega_{n} t+D_{n} \omega_{n}^{2} \sin \omega_{n} t\right] .
$$

Substituting Eqs. (3), 8a, (8b), 16a and (16c) into Eq. 10 and taking harmonic balance yields

$$
\begin{array}{r}
-m C_{n} \omega_{n}^{2}+c \omega_{n}^{q}\left(C_{n} \cos \frac{\pi q}{2}+D_{n} \sin \frac{\pi q}{2}\right)+ \\
\alpha k C_{n}+(1-\alpha) k U_{n}=A_{n}, \\
-m D_{n} \omega_{n}^{2}+c \omega_{n}^{q}\left(D_{n} \cos \frac{\pi q}{2}-C_{n} \sin \frac{\pi q}{2}\right)+ \\
\alpha k D_{n}+(1-\alpha) k V_{n}=B_{n}
\end{array}
$$

with $n=1,2, \cdots, N$. Similarly, substituting Eq.15 into Eq. 13 leads to

$$
\begin{array}{r}
\mu_{\dot{z}}=\mu_{\dot{x}}\left[A-\sqrt{\frac{2}{\pi}} \sigma_{\hat{z}}(\rho \gamma+\beta)\right]-\sqrt{\frac{2}{\pi}} \sigma_{\dot{\hat{x}}}(\gamma+\rho \beta) \mu_{z}- \\
\sqrt{\frac{2}{\pi}}\left(\frac{\gamma}{2 \sigma_{\dot{\hat{x}}}} \mu_{z} \mu_{\dot{x}}^{2}+\frac{\beta}{2 \sigma_{\hat{z}}} \mu_{\dot{x}} \mu_{z}^{2}\right) .
\end{array}
$$

Substituting Eqs.16b and (8b) into Eq. 18 leads to an equation with both sides being summation of weighted harmonics. For multi-harmonic representation of $\mu_{\dot{x}}$ and $\mu_{z}$, one may refer to 32 for an analytical solution of the weighting factors. For a special case when the periodic excitation is mono-harmonic, one may adopt an approximation that the response $\mu_{\dot{x}}$ and $\mu_{z}$ is also mono-harmonic. In this regard, consider

$$
\begin{aligned}
f(t) & =F_{0} \cos \omega_{0} t, \\
\mu_{x}(t) & =C_{0} \cos \omega_{0} t+D_{0} \sin \omega_{0} t,
\end{aligned}
$$

and

$\mu_{z}(t)=U_{0} \cos \omega_{0} t+V_{0} \sin \omega_{0} t$

for an illustrative example, where $U_{0}$ and $V_{0}$, and $C_{0}$ and $D_{0}$ are Fourier coefficients for $\mu_{z}$ and $\mu_{x}$, respectively. Eq. (18) reduces to

$-U_{0} \omega_{0} \sin \omega_{0} t+V_{0} \omega_{0} \cos \omega_{0} t=$

$\left(-C_{0} \omega_{0} \sin \omega_{0} t+D_{0} \omega_{0} \cos \omega_{0} t\right)\left[A-\sqrt{\frac{2}{\pi}} \sigma_{\hat{z}}(\rho \gamma+\beta)\right]-$

$\sqrt{\frac{2}{\pi}} \sigma_{\dot{\hat{x}}}(\gamma+\beta \rho)\left(U_{0} \cos \omega_{0} t+V_{0} \sin \omega_{0} t\right)-$

$\sqrt{\frac{2}{\pi}} \frac{\gamma}{x \sigma_{\dot{\hat{x}}}}\left(M \cos \omega_{0} t+N \sin \omega_{0} t\right)-$

$\sqrt{\frac{2}{\pi}} \frac{\beta}{2 \sigma_{z}}\left(P \cos \omega_{0} t+Q \sin \omega_{0} t\right)$
The weighting factors

$$
\begin{aligned}
& M=\frac{U_{0} C_{0}^{2} \omega_{0}^{2}}{4}-\frac{V_{0} C_{0} D_{0} \omega_{0}^{2}}{2}+\frac{3 D_{0}^{2} U_{0} \omega_{0}^{2}}{4}, \\
& N=\frac{V_{0} D_{0}^{2} \omega_{0}^{2}}{4}-\frac{U_{0} C_{0} D_{0} \omega_{0}^{2}}{2}+\frac{3 C_{0}^{2} V_{0} \omega_{0}^{2}}{4}, \\
& P=-\frac{C_{0} U_{0} V_{0} \omega_{0}}{2}+\frac{3 D_{0} U_{0}^{2} \omega_{0}}{4}+\frac{D_{0} V_{0}^{2} \omega_{0}}{4},
\end{aligned}
$$

and

$$
Q=\frac{D_{0} U_{0} V_{0} \omega_{0}}{2}-\frac{3 C_{0} V_{0}^{2} \omega_{0}}{4}-\frac{C_{0} U_{0}^{2} \omega_{0}}{4}
$$

are derived from the Fourier series of $\mu_{z} \mu_{\dot{x}}^{2}$ and $\mu_{\dot{x}} \mu_{z}^{2}$. That is

$$
\begin{array}{r}
\mu_{z} \mu_{\dot{x}}^{2}=M \cos \omega_{0} t+N \sin \omega_{0} t+(\cdots) \cos 3 \omega_{0} t+ \\
(\cdots) \sin 3 \omega_{0} t, \\
\mu_{\dot{x}} \mu_{z}^{2}=P \cos \omega_{0} t+Q \sin \omega_{0} t+(\cdots) \cos 3 \omega_{0} t+ \\
(\cdots) \sin 3 \omega_{0} t,
\end{array}
$$

where coefficients of frequency $3 \omega_{0}$ are omitted. Further taking harmonic balance on both sides of Eq. 20) gives

$$
\begin{aligned}
& -V_{0} \omega_{0}+D_{0} \omega_{0}\left[A-\sqrt{\frac{2}{\pi}} \sigma_{\hat{z}}(\rho \gamma+\beta)\right]- \\
& \sqrt{\frac{2}{\pi}} \sigma_{\dot{\hat{x}}}(\rho+\rho \beta) U_{0}-\sqrt{\frac{1}{2 \pi}}\left(\frac{\gamma}{\sigma_{\dot{\hat{x}}}} M+\frac{\beta}{\sigma_{\hat{z}}} P\right)=0, \\
& -U_{0} \omega_{0}+C_{0} \omega_{0}\left[A-\sqrt{\frac{2}{\pi}} \sigma_{\hat{z}}(\rho \gamma+\beta)\right]- \\
& \sqrt{\frac{2}{\pi}} \sigma_{\dot{\hat{x}}}(\rho+\rho \beta) V_{0}-\sqrt{\frac{1}{2 \pi}}\left(\frac{\gamma}{\sigma_{\dot{\hat{x}}}} N+\frac{\beta}{\sigma_{\hat{z}}} Q\right)=0 .
\end{aligned}
$$

Combining Eqs. 17a - 17b and Eqs. 23a - 23b yields a solution of $U_{0}, V_{0}, C_{0}$ and $D_{0}$ in terms of $\sigma_{\dot{x}}, \sigma_{\hat{z}}$ and $\rho$. However, $\sigma_{\dot{\hat{x}}}, \sigma_{\hat{z}}$ and $\rho$ are unknowns. Therefore, more relationships between the unknown Fourier coefficients and response second moments are need to supplement the set of non-linear algebraic equations shown in Eq.(17a)- 17b) and Eq. 23a)-(23b). This can be achieved by the statistical linearization method, shown in the following section. 


\section{Statistical linearization for the stochastic component}

Let $\boldsymbol{q}=\{\hat{x}, \hat{z}\}^{\mathrm{T}}$, then the equation of motion of the zero mean response $\hat{x}$ and $\hat{z}$ shown in Eqs.11 and (14) can be cast into

$\boldsymbol{M} \ddot{\boldsymbol{q}}(t)+\boldsymbol{C} \dot{\boldsymbol{q}}(t)+\boldsymbol{C}_{\boldsymbol{q}} \mathrm{D}_{\mathrm{C}}^{q} \boldsymbol{q}(t)+\boldsymbol{K} \boldsymbol{q}(t)+\boldsymbol{\Phi}(\boldsymbol{q}, \dot{\boldsymbol{q}})=\boldsymbol{Q}(t)$,

where

$$
\begin{array}{r}
\boldsymbol{M}=\left[\begin{array}{ll}
m & 0 \\
0 & 0
\end{array}\right], \boldsymbol{C}=\left[\begin{array}{ll}
0 & 0 \\
0 & 1
\end{array}\right], \boldsymbol{C}_{\boldsymbol{q}}=\left[\begin{array}{ll}
c & 0 \\
0 & 0
\end{array}\right], \boldsymbol{Q}=\left\{\begin{array}{c}
w(t) \\
0
\end{array}\right\} \\
\boldsymbol{K}=\left[\begin{array}{cc}
\alpha k(1-\alpha) k \\
0 & 0
\end{array}\right]
\end{array}
$$

are the mass, integer order damping, fractional damping and stiffness matrix, respectively; $\boldsymbol{\Phi}=\left[\phi_{1}, \phi_{2}\right]^{\mathrm{T}}$ with $\phi_{1}=0$ and

$$
\begin{aligned}
\phi_{2}= & -A \dot{\hat{x}}+\gamma\left(\hat{z}+\mu_{z}\right)\left|\dot{\hat{x}}+\mu_{\dot{x}}\right|+ \\
& \beta\left(\dot{\hat{x}}+\mu_{\dot{x}}\right)\left|\hat{z}+\mu_{z}\right|-\gamma \mathrm{E}[z|\dot{x}|]+\beta \mathrm{E}[\dot{x}|z|]
\end{aligned}
$$

is the non-linear term.

Next, according to the statistical linearization method, Eq. 24 can be equivalently written as 33.

$$
\begin{aligned}
\boldsymbol{M} \ddot{\boldsymbol{q}}(t)+\left(\boldsymbol{C}+\boldsymbol{C}_{\mathbf{e}}\right) \dot{\boldsymbol{q}}(t)+\boldsymbol{C}_{\boldsymbol{q}} \mathrm{D}_{\mathrm{C}}^{q} \boldsymbol{q}(t)+ \\
\left(\boldsymbol{K}+\boldsymbol{K}_{\mathbf{e}}\right) \boldsymbol{q}(t)=\boldsymbol{Q}(t),
\end{aligned}
$$

where

$\boldsymbol{C}_{\mathbf{e}}=\left[\begin{array}{cc}0 & 0 \\ c_{21}^{\mathrm{e}} & 0\end{array}\right]=\mathrm{E}\left[\frac{\partial \boldsymbol{\Phi}}{\partial \dot{\boldsymbol{q}}^{\mathrm{T}}}\right], \boldsymbol{K}_{\mathbf{e}}=\left[\begin{array}{cc}0 & 0 \\ 0 & k_{22}^{\mathrm{e}}\end{array}\right]=\mathrm{E}\left[\frac{\partial \boldsymbol{\Phi}}{\partial \boldsymbol{q}^{\mathrm{T}}}\right]$,

with

$c_{\mathrm{e}}=c_{21}^{\mathrm{e}}=-\mathrm{E}\left[\frac{\partial g}{\partial \dot{q}_{1}}\right], k_{\mathrm{e}}=k_{22}^{\mathrm{e}}=-\mathrm{E}\left[\frac{\partial g}{\partial q_{2}}\right]$

and

$g\left(\dot{q}_{1}, q_{2}\right)=-\phi_{2}$

Further manipulation yields

$$
\begin{aligned}
& c_{\mathrm{e}}=-\mathrm{E}\left[\frac{\partial g}{\partial \dot{q}_{1}}\right]=-A+\gamma \mathrm{E}[z \operatorname{sgn}(\dot{x})]+\beta \mathrm{E}[|z|], \\
& k_{\mathrm{e}}=-\mathrm{E}\left[\frac{\partial g}{\partial q_{2}}\right]=\gamma \mathrm{E}[|\dot{x}|]+\beta \mathrm{E}[\dot{x} \operatorname{sgn}(z)] .
\end{aligned}
$$

The expectation of non-linear functions in Eq. 27a27b can be approximated as 30.

$$
\begin{aligned}
\mathrm{E}[z \operatorname{sgn}(\dot{x})] & =\sqrt{\frac{2}{\pi}} \rho \sigma_{z}\left(1-\frac{\mu_{\dot{x}}^{2}}{2 \sigma_{\dot{\hat{x}}}^{2}}\right)+\sqrt{\frac{2}{\pi}} \frac{\mu_{z} \mu_{\dot{x}}}{\sigma_{\dot{\hat{x}}}}, \\
\mathrm{E}[\dot{x} \operatorname{sgn}(z)] & =\sqrt{\frac{2}{\pi}} \rho \sigma_{\dot{\hat{x}}}\left(1-\frac{\mu_{z}^{2}}{2 \sigma_{\hat{z}}^{2}}\right)+\sqrt{\frac{2}{\pi}} \frac{\mu_{z} \mu_{\dot{x}}}{\sigma_{\hat{z}}}, \\
\mathrm{E}[|z|] & =\sqrt{\frac{2}{\pi}} \sigma_{\hat{z}}\left(1-\frac{\mu_{z}^{2}}{2 \sigma_{\hat{z}}^{2}}\right)+\sqrt{\frac{2}{\pi}} \frac{\mu_{z}^{2}}{\sigma_{\hat{z}}} \\
\mathrm{E}[|\dot{x}|] & =\sqrt{\frac{2}{\pi}} \sigma_{\dot{\hat{x}}}\left(1-\frac{\mu_{\dot{x}}^{2}}{2 \sigma_{\dot{\hat{x}}^{2}}}\right)+\sqrt{\frac{2}{\pi}} \frac{\mu_{\dot{\dot{x}}}^{2}}{\sigma_{\dot{\hat{x}}}}
\end{aligned}
$$

Combining Eqs.28a-28d with Eqs.27a-27b one may find that the equivalent parameters of the system are time varying with frequency $\omega_{0}$, for the mean process $\mu_{\dot{x}}$ and $\mu_{z}$ are included. Note that the standard deviations $\sigma_{z}$ and $\sigma_{\dot{\hat{x}}}$ are cyclostationary when $t \rightarrow \infty$. Therefore, the fast varying content can be eliminated by averaging over the fundamental period $T_{0}=2 \pi / \omega_{0}$. That is

$$
\bar{c}_{\mathrm{e}}=\frac{\gamma}{T_{0}} \int_{0}^{T_{0}} \mathrm{E}[z \operatorname{sgn}(\dot{x})] \mathrm{d} t+\frac{\beta}{T_{0}} \int_{0}^{T_{0}} \mathrm{E}[|z|] \mathrm{d} t-A,
$$

$\bar{k}_{\mathrm{e}}=\frac{\gamma}{T_{0}} \int_{0}^{T_{0}} \mathrm{E}[|\dot{x}|] \mathrm{d} t+\frac{\beta}{T_{0}} \int_{0}^{T_{0}} \mathrm{E}[\dot{x} \operatorname{sgn}(z)] \mathrm{d} t$,

where the deterministic averaging on the right hand side of Eq.22a -29b yields the following approximations 30

$$
\begin{aligned}
& \mathrm{E}[z \operatorname{sgn}(\dot{x})] \approx \sqrt{\frac{2}{\pi}} \rho \sigma_{\hat{z}}\left[1-\frac{\left(C_{0}^{2}+D_{0}^{2}\right) \omega_{0}^{2}}{4 \sigma_{\dot{\hat{x}}}^{2}}\right]+ \\
& \sqrt{\frac{2}{\pi} \frac{\left(D_{0} U_{0}-C_{0} V_{0}\right) \omega_{0}}{2 \sigma_{\dot{\hat{x}}}},} \\
& \mathrm{E}[\dot{x} \operatorname{sgn}(z)] \approx \sqrt{\frac{2}{\pi} \rho \sigma_{\dot{\hat{x}}}\left[1-\frac{U_{0}^{2}+V_{0}^{2}}{4 \sigma_{\hat{z}}^{2}}\right]+} \\
& \mathrm{E}[|z|] \approx \sqrt{\frac{2}{\pi}} \sigma_{\hat{z}}\left[1-\frac{\left.U_{0}^{2}+D_{0} U_{0}-C_{0} V_{0}\right) \omega_{0}}{4 \sigma_{\hat{z}}^{2}},\right. \\
& \mathrm{E}[|\dot{x}|] \approx \sqrt{\frac{2}{\pi}} \sigma_{\dot{\hat{x}}}\left[1+\frac{\left(C_{0}^{2}+D_{0}^{2}\right) \omega_{0}^{2}}{4 \sigma_{\dot{x}^{2}}}\right] .
\end{aligned}
$$

Note that the approximate equivalent parameters $\bar{c}_{\mathrm{e}}$ and $\bar{k}_{\mathrm{e}}$ depend on seven unknowns $C_{0}, D_{0}, U_{0}, V_{0}, \sigma_{\dot{\hat{x}}}, \sigma_{\hat{z}}$ and $\rho$.

Further, the supplemented relationship between the unknown response statistics $\sigma_{\dot{\hat{x}}}, \sigma_{\hat{z}}$ and $\rho$, and the Fourier coefficients of the deterministic response component, 
can be obtained by the standard linear random vibration theory for linear dynamic systems with fractional elements 34. In this regard,

$\boldsymbol{S}_{\boldsymbol{q}}(\omega)=\boldsymbol{H}(\omega) \boldsymbol{S}_{\boldsymbol{Q}}(\omega) \boldsymbol{H}^{\mathrm{T}}(\omega)$

where

$\boldsymbol{S}_{\boldsymbol{Q}}(\omega)=\left[\begin{array}{cr}S_{w}(\omega) & 0 \\ 0 & 0\end{array}\right], \boldsymbol{S}_{\boldsymbol{q}}=\left[\begin{array}{cc}S_{\hat{x} \hat{x}} & S_{\hat{x} \hat{z}} \\ S_{\hat{z} \hat{x}} & S_{\hat{z} \hat{z}}\end{array}\right]$

is the two-sided PSD matrix of $\boldsymbol{Q}$ and $\boldsymbol{q}$, respectively;

$\boldsymbol{H}(\omega)=1 /\left(-\omega^{2} \boldsymbol{M}+\mathrm{i} \omega\left(\boldsymbol{C}+\boldsymbol{C}_{e}\right)+\left(\boldsymbol{K}+\boldsymbol{K}_{e}\right)+(\mathrm{i} \omega)^{q} \boldsymbol{C}_{\boldsymbol{q}}\right.$

is the frequency response matrix. $S(\omega)$ is the PSD of the stochastic excitation component; $S_{\hat{x} \hat{x}}, S_{\hat{x} \hat{z}}=S_{\hat{z} \hat{x}}$ and $S_{\hat{z} \hat{z}}$ are the two-sides auto-PSD of $\hat{x}$, the two-sides cross-PSD of $(\hat{x}, \hat{z})$, and the two-sides auto-PSD of $\hat{z}$. Therefore,

$$
\begin{aligned}
& \sigma_{\hat{\hat{x}}}^{2}=\int_{-\infty}^{\infty} \omega^{2} S_{\hat{x} \hat{x}}(\omega) \mathrm{d} \omega, \\
& \sigma_{\hat{z}}^{2}=\int_{-\infty}^{\infty} S_{\hat{z} \hat{z}}(\omega) \mathrm{d} \omega, \\
& \rho \sigma_{\hat{\hat{x}}} \sigma_{\hat{z}}=-\int_{-\infty}^{\infty} \mathrm{i} \omega S_{\hat{x} \hat{z}}(\omega) \mathrm{d} \omega .
\end{aligned}
$$

\section{Implementation procedures}

To summarize, the harmonic balance method for the deterministic response component leads to four indeterminate non-linear algebraic equations 17a - 17b and (23a)-23b). These equations include seven unknowns $\left(C_{0}, D_{0}, U_{0}, V_{0}, \sigma_{\dot{\hat{x}}}, \sigma_{\hat{z}}, \rho\right)$ that only four equations do not yields an unique solution. Besides, Eqs. (32a)- (32c) establish supplementary relationships between the unknown response Fourier coefficients $\left(C_{0}, D_{0}, U_{0}, V_{0}\right)$ and the response statistics $\left(\sigma_{\dot{\hat{x}}}, \sigma_{\hat{z}}, \rho\right)$. Therefore, the procedures of the proposed method is the following:

1. Consider the corresponding fractional-order linear system $\left(F_{0}=0, \alpha=0\right)$ subjected to the stochastic excitation. Set the initial guess of the response variances equal to those of the fractional-order linear system. Set the zero-mean hysteretic displacement $\hat{z}=\hat{x}$, and thus, $\rho=0$.

2. Solve Eq. 17a)-17b and Eq. (23a)-23b simultaneously to obtain the first guess of $C_{0}, D_{0}, U_{0}$ and $V_{0}$, using standard numerical schemes for non-linear algebraic equations. Newton's iterative method is used in this paper.

3. Use Eq. 27a - $27 \mathrm{~b}$ and Eq. (30a)-Eq. (30d) to get $c_{\mathrm{e}}$ and $k_{\mathrm{e}}$. Use Eq.(31) and Eq.(32a)-(32c) to update $\sigma_{\dot{\hat{x}}}, \sigma_{\hat{z}}$ and $\rho$.
4. Repeat Step 1 to Step 3 until certain convergence criterion is achieved.

For applying Newton's iterative method in Step 2, one needs to solve the following matrix equation for the unknown Fourier coefficients of the harmonic component. That is

$\boldsymbol{K}\left(\boldsymbol{\alpha}^{(i)}\right)+\boldsymbol{J}\left(\boldsymbol{\alpha}^{(i)}\right)\left(\boldsymbol{\alpha}^{(i+1)}-\boldsymbol{\alpha}^{(i)}\right)=0$

where the superscript $(i)$ denotes the $i$ th iteration step $\boldsymbol{\alpha}$ is the vector of the unknowns $\boldsymbol{\alpha}=\left[C_{0}, D_{0}, U_{0}, V_{0}\right]^{\mathrm{T}}$; The colum vector $\boldsymbol{K}$ with four entries can be written

$$
\begin{array}{r}
\boldsymbol{K}_{1}(\boldsymbol{\alpha})=-m C_{0} \omega_{0}^{2}+c \omega_{0}^{q}\left(C_{0} \cos \frac{\pi q}{2}+D_{0} \sin \frac{\pi q}{2}\right)+ \\
\alpha k C_{0}+(1-\alpha) k U_{0}
\end{array}
$$

$$
\begin{array}{r}
\boldsymbol{K}_{2}(\boldsymbol{\alpha})=-m D_{0} \omega_{0}^{2}+c \omega_{0}^{q}\left(D_{0} \cos \frac{\pi q}{2}-C_{0} \sin \frac{\pi q}{2}\right)+ \\
\alpha k D_{0}+(1-\alpha) k V_{0}
\end{array}
$$

$$
\begin{array}{r}
\boldsymbol{K}_{3}(\boldsymbol{\alpha})=-V_{0} \omega_{0}+D_{0} \omega_{0}\left[A-\sqrt{\frac{2}{\pi}} \sigma_{\hat{z}}(\rho \gamma+\beta)\right]- \\
\sqrt{\frac{2}{\pi}} \sigma_{\dot{\hat{x}}}(\rho+\rho \beta) U_{0}-\sqrt{\frac{1}{2 \pi}}\left(\frac{\gamma}{\sigma_{\hat{\hat{x}}}} M+\frac{\beta}{\sigma_{\hat{z}}} P\right) \\
\boldsymbol{K}_{4}(\boldsymbol{\alpha})=-U_{0} \omega_{0}+C_{0} \omega_{0}\left[A-\sqrt{\frac{2}{\pi}} \sigma_{\hat{z}}(\rho \gamma+\beta)\right]- \\
\sqrt{\frac{2}{\pi}} \sigma_{\dot{\hat{x}}}(\rho+\rho \beta) V_{0}-\sqrt{\frac{1}{2 \pi}}\left(\frac{\gamma}{\sigma_{\hat{\hat{x}}}} N+\frac{\beta}{\sigma_{\hat{z}}} Q\right)
\end{array}
$$

the Jacobin matrix is

$\boldsymbol{J}=\frac{\partial \boldsymbol{K}}{\partial \boldsymbol{\alpha}^{\mathrm{T}}}$

\section{Numerical example}

A Bouc-Wen hysteretic model endowed with fractional element and subjected to combined harmonic and stochastic excitation (white/colored) is considered, where the parameters of the corresponding integer-order linear system $m=1, \omega_{n}=\sqrt{\frac{k}{m}}=1, \zeta=\frac{c}{2 m \omega_{n}}=0.1$ are used.

\subsection{White noise as the stochastic excitation}

The strength of the white noise excitation is chosen as $w(t)=\sqrt{4 \zeta} f_{n}(t)$, where $f_{n}(t)$ has 'unit strength', i.e., 
$\mathrm{E}\left[f_{n}(t) f_{n}(t+\tau)\right]=\delta(\tau)$. Dividing both sides of Eq. (1) by $m$ yields

$$
\begin{aligned}
\ddot{x}(t)+2 \zeta \omega_{n}^{2-q} \mathrm{D}_{\mathrm{C}}^{q}[x(t)]+\alpha \omega_{n}^{2} x(t)+ \\
(1-\alpha) \omega_{n}^{2} z(t)=w(t)+F_{0} \sin \omega_{0} t
\end{aligned}
$$

When $\alpha=1, F_{0}=0, q=1$ the fractional-order hysteretic system reduces to a special case of the linear system with integer-order derivative and subjected to stochastic excitation only. The displacement standard deviation of the reduced linear system is $\sigma_{\hat{x}}=\sigma_{x}=1$.

In this illustrative case, to demonstrate the applicability of the proposed method, the amplitude and the frequency of the harmonic excitation is chosen as $F_{0}=1$ and $\omega_{0}=1.2$, respectively. Both softening and hardening Bouc-Wen systems with fractional order $q=0.5$ are considered herein. For the softening Bouc-Wen system, the hysteretic parameters are $A=1, \gamma=0.5, \beta=$ $0.5, n=1, \alpha=0.1$, whereas for the hardening BoucWen system, all the other hysteretic parameters are the same as in the softening case except $\beta=-0.35, \gamma=$ 0.65. The response comparison shown in Figs 1(a) 1(b) for the softening Bouc-Wen model verifies the accuracy of the proposed method. Specifically, the deterministic component time history and the stochastic component standard deviation of the displacement $x(t)$, obtained by the proposed method is in good agreement with the pertinent Monte Carlo estimates over 10,000 samples. Besides, it seems that the proposed method yields a stationary standard deviation of the stochastic component, vis-a-vis the MC estimates gives a cyclostationary standard deviation. This is caused by the coupling effect between the deterministic and random response component, shown in Eqs. (28a)-(28d). These equations indicate that the equivalent parameters, and thus, the standard deviation of the stochastic component, depend on deterministic component. However, in the proposed analytical method, the oscillation of the equivalent parameters, and thus, of the cyclostationary variance, is eliminated by averaging over one period for further simplification (see Eqs. 29a)-(29b)). Therefore, the response variance during the cyclostationary phase obtained by the Monte Carlo data is averaged for the ensuing error analysis. Specifically, for the deterministic response $\mu_{x}(t)$ shown in Fig. 1(a), the response amplitudes obtained by the proposed method is around $8.89 \%$ less than the Monte Carlo estimates, whereas for the stationary standard deviation shown in Fig 1(b), the analytical result is about $11.68 \%$ less the MC data. Further MC investigations show that the amplitude of harmonic-like cyclostationary part of the standard deviation depends on the amplitude and the frequency of the harmonic excitation. Besides, it seems that the oscillation frequency of the cyclostationary standard devi- ation is twice as higher as the excitation frequency. This conclusion is reasonable because the equivalent stiffness, shown in Eq. 27b), depends on quadratic terms of the deterministic response component.

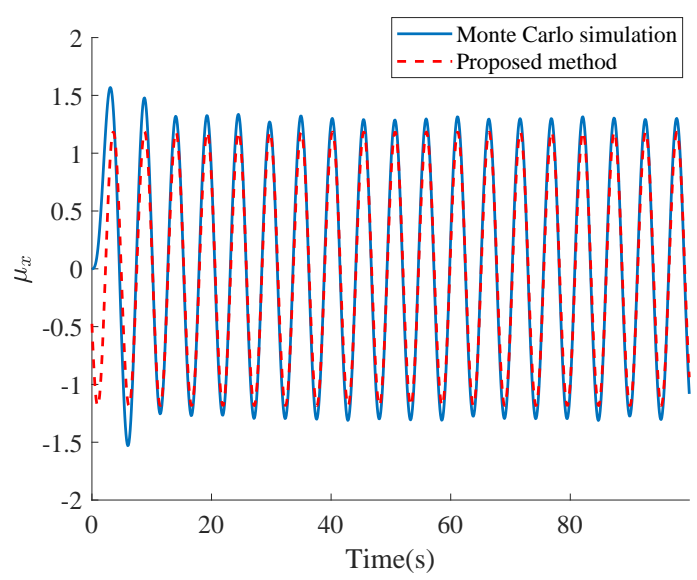

(a) Mean response $\mu_{x}$

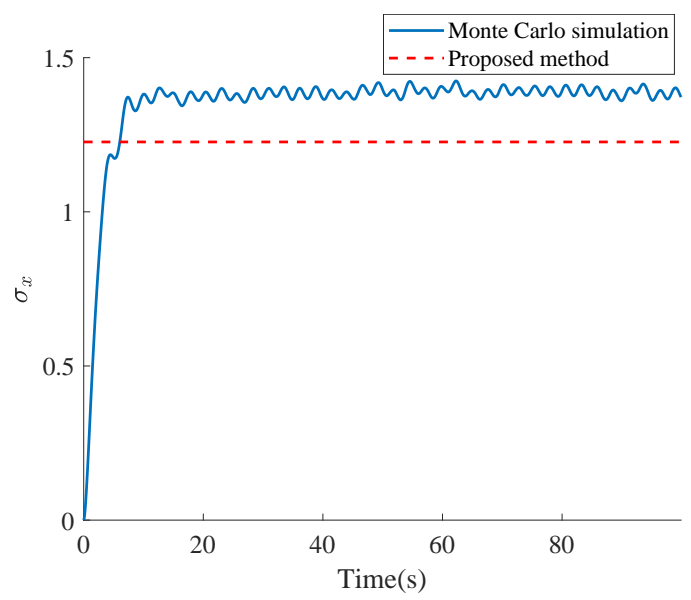

(b) $\operatorname{Std} \sigma_{\hat{x}}$

Fig. 1 Displacement of the softening Bouc-Wen system subjected to combined excitation.

Numerical investigations, as shown in Figs. 2(a). 2(b) demonstrate the applicability of the proposed method for the considered hardening Bouc-Wen model endowed with fractional element. Error analysis suggests satisfactory accuracy of the proposed method. Specifically, for the displacement response $x(t)$, the amplitude of the deterministic component and the standard deviation of the stochastic component, obtained by the proposed method, is $16.55 \%$ and $10.5 \%$ lower than the corresponding MC estimates, respectively. All the above errors are within the reasonable range of the classic statistical linearization method reported by previous researches 33 . 


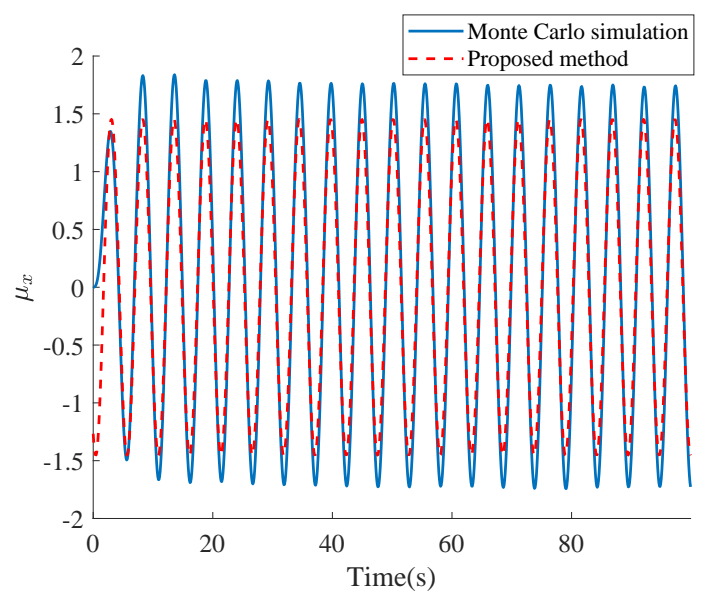

(a) Mean response $\mu_{x}$

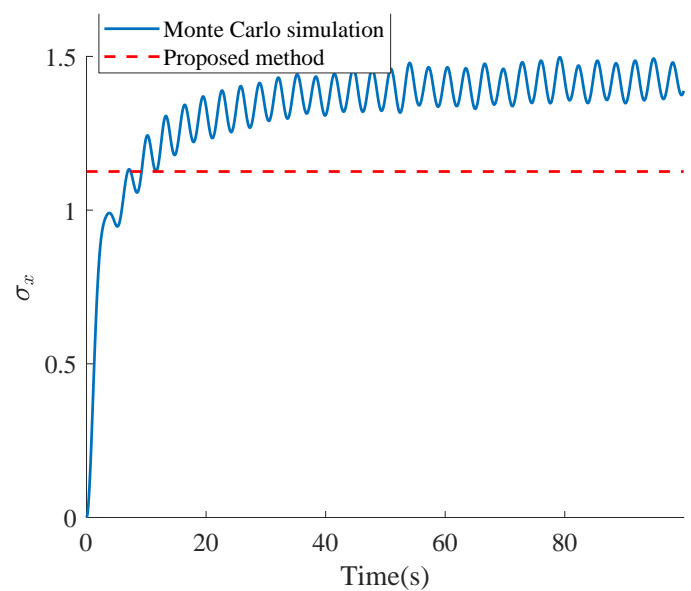

(b) $\operatorname{Std} \sigma_{\hat{x}}$

Fig. 2 Displacement of the hardening Bouc-Wen system subjected to combined excitation.

Sample hysteretic loops of the considered softening and hardening Bouc-Wen system endowed with fractional element under combined harmonic and white noise excitation is shown in Figs 3(a) 3(b) respectively. It is seen that for both circumstances, the hysteretic loops exhibit history-depended strong non-linearity. Besides, compared to the hysteretic loops under harmonic excitation, the white noise disturbance renders the loop center move around the equilibrium position.

The ensuing analyses further investigate the applicability of the proposed method under other harmonic excitation with different frequencies. Consider the softening Bouc-Wen model with the same parameters as in Fig. 3(a), Fig 4 shows the response standard deviation $\left(\sigma_{\hat{x}}, \sigma_{\hat{x}}, \sigma_{\hat{z}}\right)$ versus excitation frequency curve when the harmonic excitation amplitude is $F_{0}=1$. It seems that the displacement standard deviation obtained by the proposed method agrees well with the Monte Carlo estimates over 10,000 sample responses.

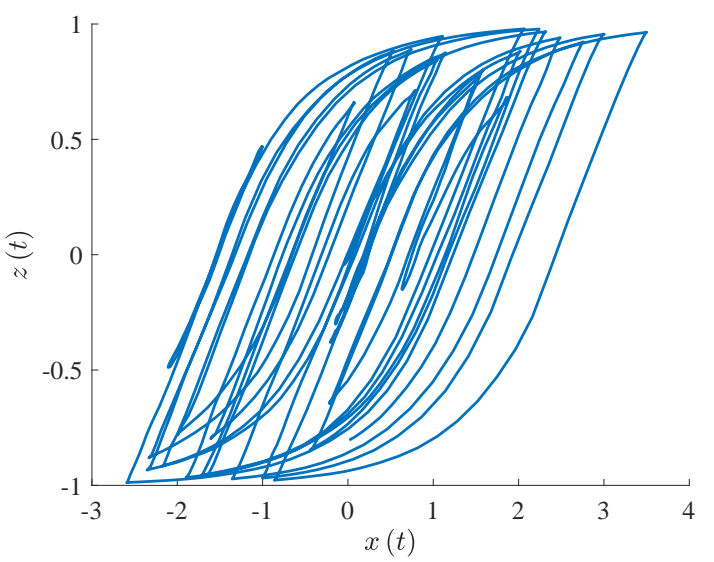

(a) Softening hysteretic loop

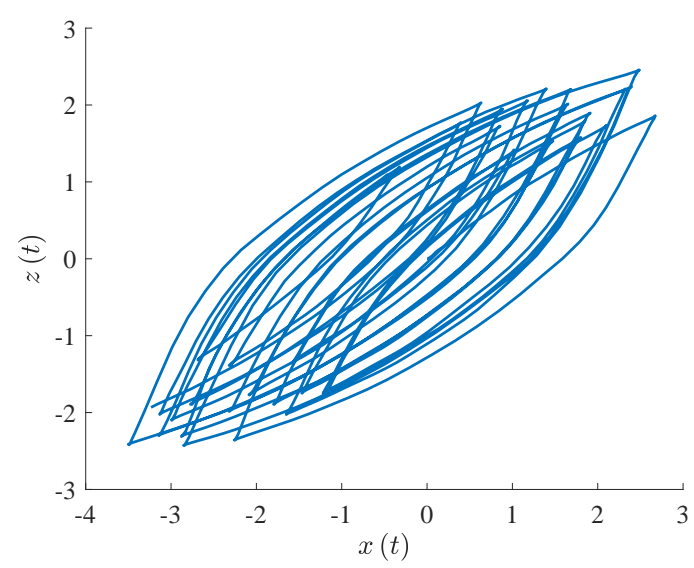

(b) Hardening hysteretic loop

Fig. 3 Hysteretic loops of the Bouc-Wen dynamic system subjected to combined harmonic and stochastic excitation.

The amplitudes of the deterministic response component $\left(\mu_{x}, \mu_{\dot{x}}, \mu_{z}\right)$ for different excitation frequencies, obtained by the proposed method and the pertinent Monte Carlo simulation, are compared in Fig 5 when $F_{0}=1$. It seems that under the considered cases, the amplitudes of the deterministic component obtained by the proposed method are in good agreement with the Monte Carlo estimates over 10,000 sample responses. Further investigations on the applicability and accuracy of the proposed method for response of the hardening Bouc-Wen model further validate the versatility of this method. These results are not included herein for they indicate similar conclusions.

Investigate next the applicability of the proposed method for the considered softening/hardening BoucWen systems with different fractional orders. The amplitude and frequency of harmonic excitation are selected to be $F_{0}=1$ and $\omega_{0}=1.2$, while both softening and hardening system with the same hysteretic param- 


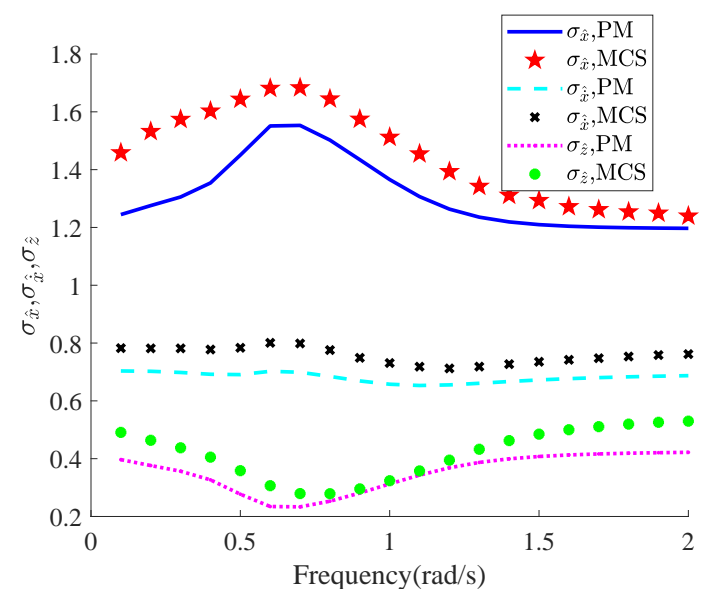

Fig. 4 Standard deviation of the stochastic response component of a softening Bouc-Wen system subjected to combined stochastic excitation and harmonic excitation with different frequencies

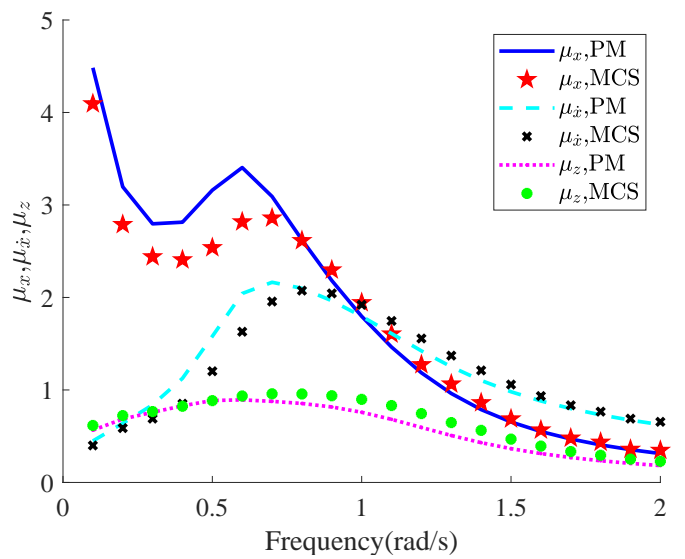

Fig. 5 Amplitude of the deterministic response component of a softening Bouc-Wen system subjected to combined stochastic excitation and harmonic excitation with different frequencies

eters as in Figs. 3(a) 3(b) are considered herein for illustration. Figs. 6(a) 6(b) show the response standard deviation $\left(\sigma_{\hat{x}}, \sigma_{\dot{\hat{x}}}, \sigma_{\hat{z}}\right)$ versus fractional order curve for the softening and for the hardening system, respectively. It seems that for the considered cases with fractional order $q \in[0.1,0.9]$, the response statistical characteristics obtained by the proposed method agrees quite well with the pertinent Monte Carlo estimates. Comparisons regarding the fractional-order versus deterministic response amplitude curve is not included here for they indicate similar conclusions.

\subsection{Colored noise as the stochastic excitation}

The proposed method can be extended for response determination of a hysteretic systems endowed with frac-

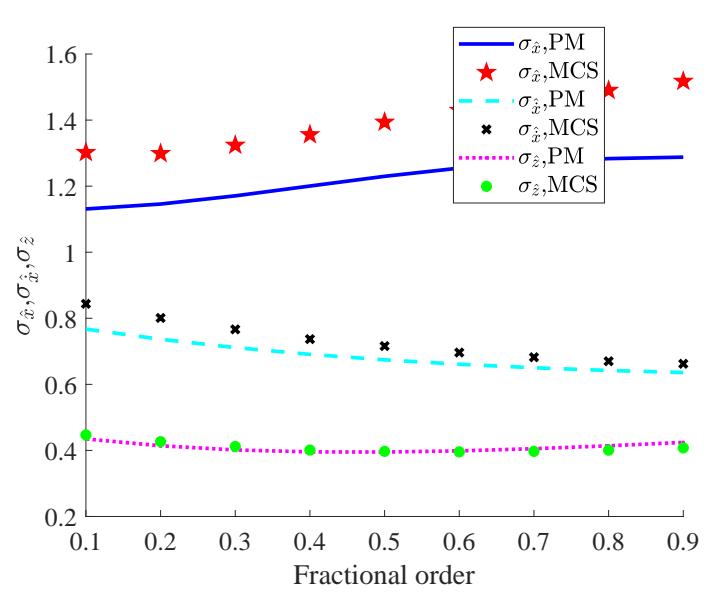

(a) softening system

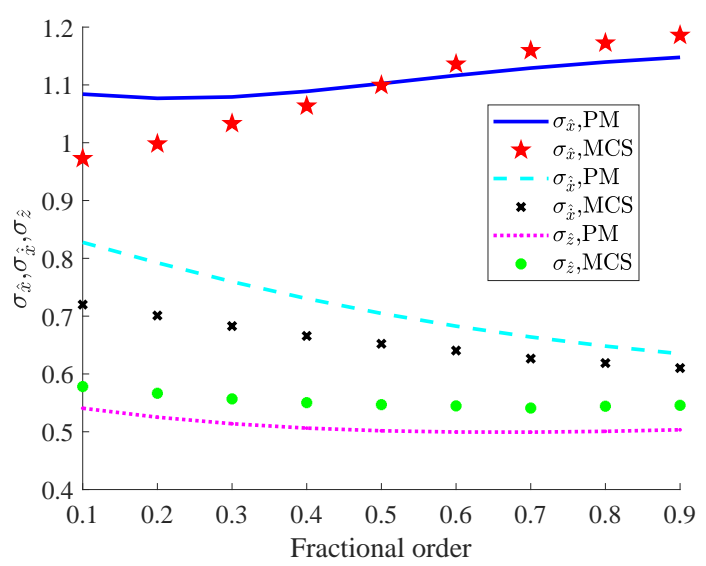

(b) hardening system

Fig. 6 Standard deviation of the stochastic response component of softening and hardening Bouc-Wen systems subjected to combined stochastic excitation and harmonic excitation with different fractional orders

tional element and subjected to combined periodic and colored noise, readily. Consider the equation of motion shown in Eq. (36), with $w(t)$ being a zero-mean colored noise with

$S_{w}(\omega)=\frac{1+4 \xi_{\mathrm{g}}^{2} \frac{\omega^{2}}{\omega_{\mathrm{g}}^{2}}}{\left(1-\frac{\omega^{2}}{\omega_{\mathrm{g}}^{2}}\right)^{2}+4 \xi_{\mathrm{g}}^{2} \frac{\omega^{2}}{\omega_{\mathrm{g}}^{2}}} \cdot S_{0}$

where $\zeta_{\mathrm{g}}=0.2$ and $\omega_{\mathrm{g}}=2$ is the damping ratio and natural frequency for the filter; $S_{0}=2 \zeta / \pi$ is the power spectral density for the white noise to be filtered. In this illustrative case, to demonstrate the applicability of the proposed method, the amplitude and the frequency of the harmonic excitation is chosen as $F_{0}=1$ and $\omega_{0}=1$, respectively.

Consider both a softening and a hardening BoucWen system. For the softening Bouc-Wen system, the hysteretic parameters are $A=1, \gamma=0.5, \beta=0.5$, 
$n=1, \alpha=0.1$, whereas for hardening Bouc-Wen system, all the other hysteretic parameters are the same as in the softening case except $\beta=-0.1, \gamma=0.8$. The response comparison shown in Figs $7(\mathrm{a})$. $7(\mathrm{~b})$ for the softening Bouc-Wen system verifies the accuracy of the proposed method. Specifically, for the deterministic harmonic response $x(t)$ shown in Fig. 7(a), the amplitude of the deterministic response component, obtained by the proposed method is around $3.83 \%$ less than the Monte Carlo estimates. For the stationary standard deviation of the stochastic response component calculated by the proposed method, shown in Fig 7(b), is about $11.63 \%$ less than the time average of the cyclostationary standard deviation obtained by the Monte Carlo simulation. Further MC investigations show that the amplitude of the harmonic-like cyclostationary part of the standard deviation depends on the amplitude and the frequency of the harmonic excitation.

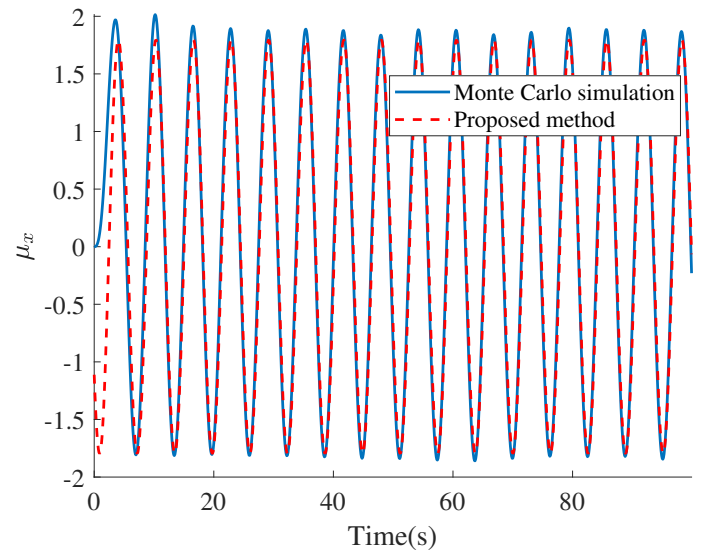

(a) Mean response $\mu_{x}$

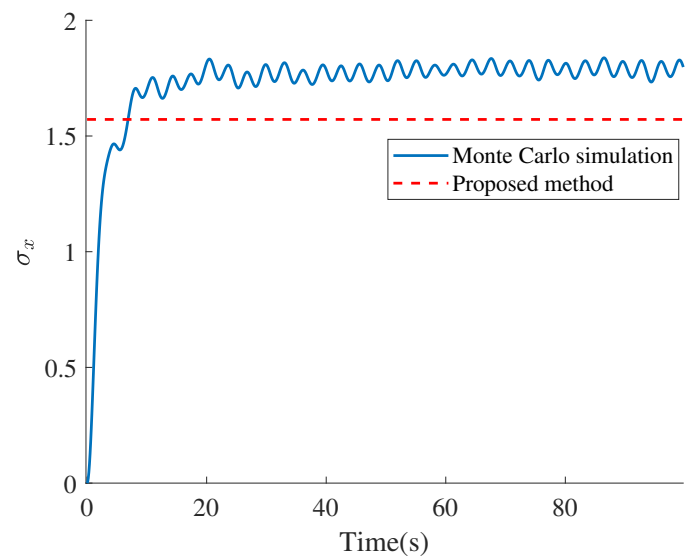

(b) $\operatorname{Std} \sigma_{\hat{x}}$

Fig. 7 Displacement of the softening Bouc-Wen system subjected to combined excitation.
Numerical investigations, as shown in Figs. 8(a). 8(b) demonstrate the applicability of the proposed method for the considered hardening Bouc-Wen system endowed with fractional elements. Error analysis suggests satisfactory accuracy of the proposed method. Specifically, for the displacement response $x(t)$, the amplitude of the deterministic component and the standard deviation of the stochastic component, obtained by the proposed method, is $11.32 \%$ and $5.91 \%$ less than the corresponding $\mathrm{MC}$ estimates, respectively. All the above errors are within the reasonable range of the classic statistical linearization method.

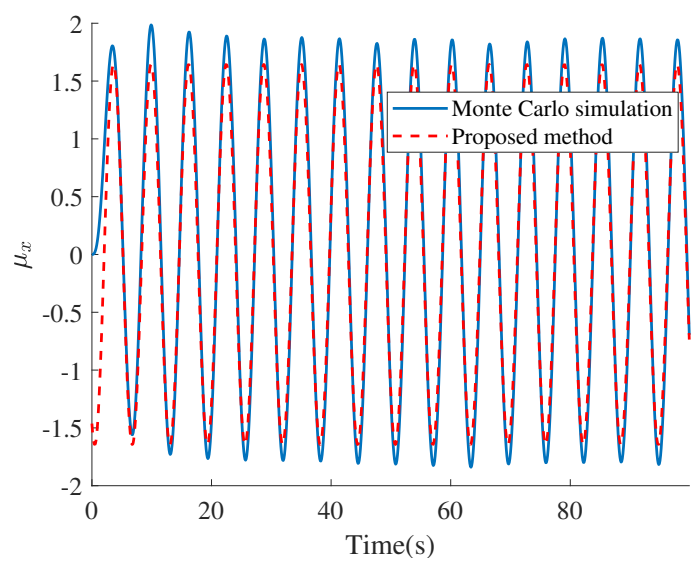

(a) Mean response $\mu_{x}$

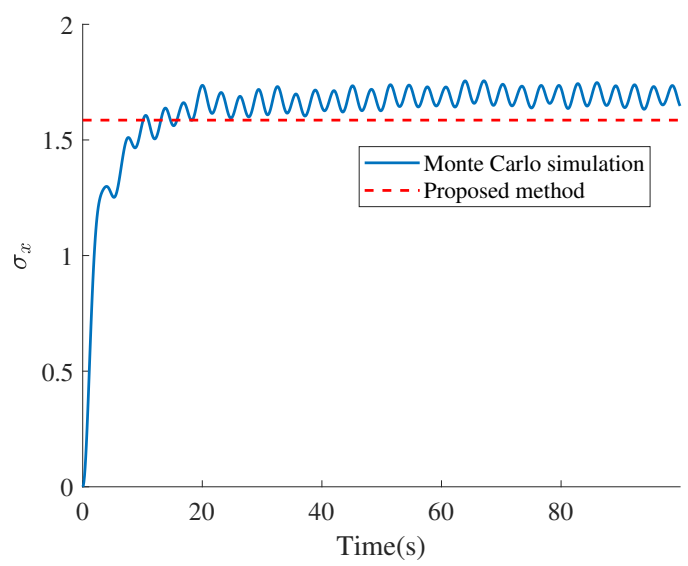

(b) $\operatorname{Std} \sigma_{\hat{x}}$

Fig. 8 Displacement of the hardening Bouc-Wen system subjected to combined excitation.

The same conclusion as in the white noise case can be obtained, that the sample hysteretic loops of the considered hysteretic system under combined harmonic and colored noise excitation, exhibit history depended strong non-linearity. Besides, compared to the hysteretic loops under harmonic excitation, the colored noise dis- 
turbance renders the loop center move around the equilibrium position.

The ensuing analysis further investigates the applicability of the proposed method under other harmonic excitations with different frequencies. Consider the softening Bouc-Wen model with the same parameters as in Figs. 7(a) 7(b) Fig. 9 shows the response standard deviation $\left(\sigma_{\hat{x}}, \sigma_{\dot{\hat{x}}}, \sigma_{\hat{z}}\right)$ versus excitation frequency curve, when the harmonic excitation amplitude is $F_{0}=1$. It seems that the proposed linearization method exhibits reasonable accuracy $(<18 \%)$ over all the considered excitation frequencies for the derived displacement standard deviation compared with the Monte Carlo estimates.

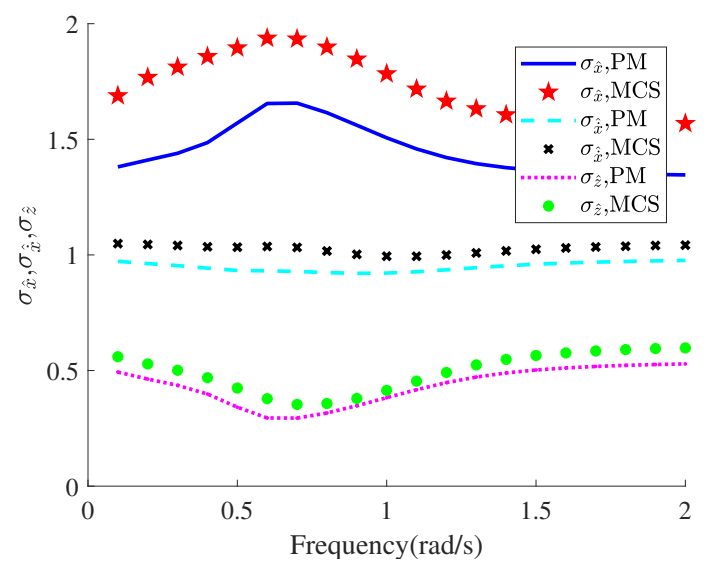

Fig. 9 Standard deviation of the stochastic response component of a softening Bouc-Wen system subjected to combined stochastic excitation and harmonic excitation with different frequencies

The amplitudes of the deterministic response component for the harmonic excitation with different frequencies, obtained by the proposed method and estimated by the pertinent Monte Carlo simulation, are compared in Figs. 10 when $F_{0}=1$. It seems that under the considered cases, the amplitudes of the deterministic response component versus harmonic excitation frequency curve, can be well predicted by the proposed analytical method.

Investigations on the applicability and accuracy of the proposed method regarding the amplitude-frequency curve of the deterministic responses of the considered hardening Bouc-Wen system, further validate the similar conclusions as in the softening Bouc-Wen case, which are not included herein for the limited space. All the preceding numerical examples yield results with reasonable accuracy when the relevant assumptions 30. are not violated.

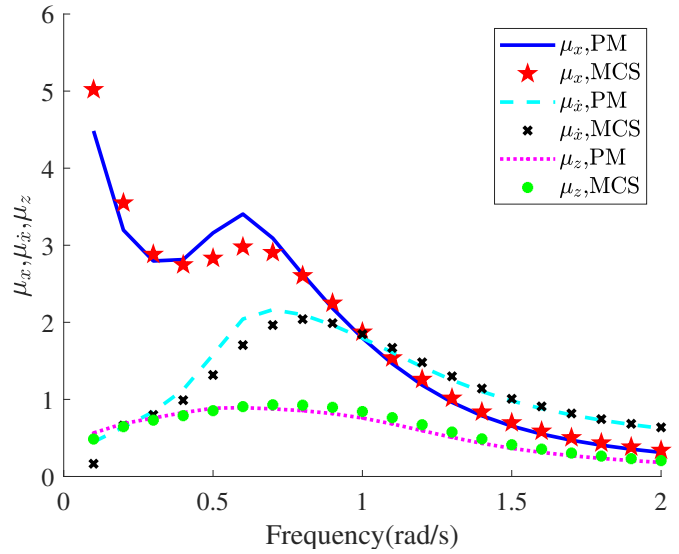

Fig. 10 Amplitude of the deterministic response component of a softening Bouc-Wen system subjected to combined stochastic excitation and harmonic excitation with different frequencies

The last analysis investigates the applicability of the proposed method for the considered Bouc-Wen systems endowed with different fractional orders. The amplitude and frequency of harmonic excitation are selected to be $F_{0}=1$ and $\omega_{0}=1$, while both softening and hardening system with the same parameters as in Figs. $7(\mathrm{a}) \mathrm{7(b)}$ and $8(\mathrm{a}) \mathrm{8( \textrm {b } )}$, respectively, are considered herein for illustration. Figs. 11(a) 11(b) show the response standard deviation $\left(\sigma_{\hat{x}}, \sigma_{\dot{\hat{x}}}, \sigma_{\hat{z}}\right)$ versus fractional order curve for softening and hardening system, respectively. It seems that for the considered cases, the response statistical characteristics obtained by the proposed method show reasonable accuracy when compared with the pertinent Monte Carlo estimates.

\section{Concluding remarks}

A statistical linearization method has been proposed for the response determination of a SDOF hysteretic system endowed with fractional element and subjected to combined periodic and white/colored excitation. This has been achieved by decomposing the total stochastic response into a summation of deterministic and zeromean stochastic component. By doing so, the differential equation governing the total response has been decomposed into two sets of differential equations, governing the deterministic response component and the zero-mean stochastic response component, respectively. Next, the harmonic balance method has been utilized to establish a relationship between the Fourier coefficients of the periodic excitation and those of the deterministic response component, whereas the statistical linearization has been used for the second moments of the stochastic response component. However, the de- 


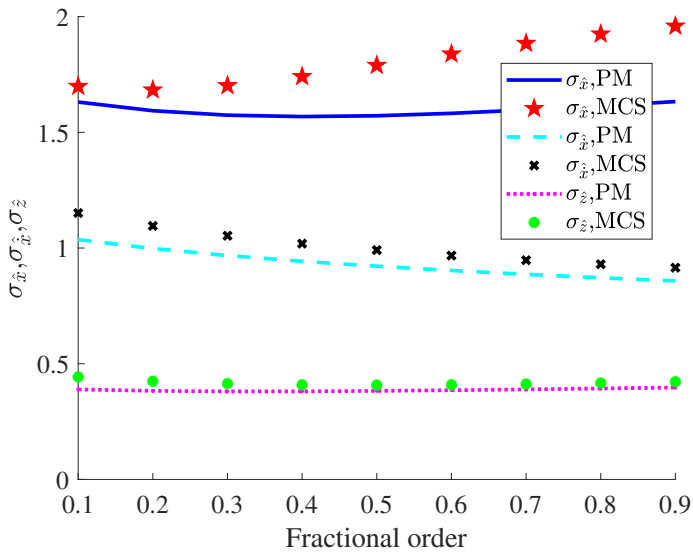

(a) softening system

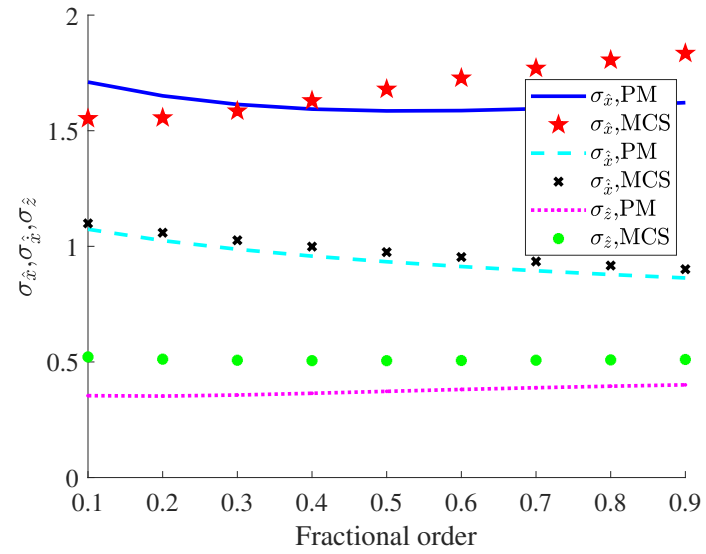

(b) hardening system

Fig. 11 Standard deviation of the stochastic response component of softening and hardening Bouc-Wen systems subjected to combined stochastic excitation and harmonic excitation with different fractional orders

rived non-linear algebraic equations obtained by the two techniques are coupled, and thus standard numerical iteration method has been adopted to obtain the response quantities numerically and simultaneously. Finally, the pertinent Monte Carlo simulation has been used to demonstrate the applicability and accuracy of the proposed method. Specifically, a softening and hardening Bouc-Wen hysteretic system endowed with fractional element and subjected to combined excitation has been used as numerical examples for illustration. It has been found that the accuracy of the proposed method is comparable with the well established statistical linearization method for a integer-order Bouc-Wen system subjected to stochastic excitation only. It should be noted that the proposed method can be served as an beneficial attempt toward efficient and versatile solution for the considered problem. Further investigation should focus on the extension of the method for multi-degree-of-freedom fractional-order systems with other type of hysteretic models and subjected to nonstationary colored excitation.

\section{Acknowledgment}

This work was supported by the National Natural Science Foundation of China (Grant no. 52078399). The first author would like to thank the Chinese Scholarship Council (CSC) for financial support (File no. 201706955030) during his visit to Rice University as a visiting scholar. The first author would like to greatly thank Professor Pol D. Spanos at Rice University for the discussing the statistical linearization method for fractional-order systems.

\section{Declarations}

- Funding: All sources of funding for the research reported have been declared.

- Competing of interest: The authors declare that they have no competing interests.

- Code availability: All codes relevant to the present work are available upon request.

- Authors' contributions: KF conceived the study, derived the theoretical formulation and finalized the manuscript. HRJ composed the relevant codes, drafted the manuscript. ZYJ participated in coding, and drafting the manuscript.

- Availability of data and material: Not applicable.

\section{References}

1. Hong SR, Choi SB, Choi YT, Wereley NM (2005) A hydro-mechanical model for hysteretic damping force prediction of er damper: experimental verification. Journal of Sound \& Vibration 285(45):1180-1188

2. Okuizumi N, Kimura K (2004) Multiple time scale analysis of hysteretic systems subjected to harmonic excitation. Journal of Sound \& Vibration $272(3-5): 675-701$

3. Iwan, W D (1968) Response of the bilinear hysteretic system to stationary random excitation. The Journal of the Acoustical Society of America $43(3): 545-552$

4. Caughey TK (1960) Sinusoidal Excitation of a System With Bilinear Hysteresis. Journal of Applied Mechanics 27(4):640-643

5. Bouc R (1967) Forced vibration of mechanical systems with hysteresis. In: Proceedings of the Fourth Conference on Nonlinear Oscillation, Prague 
6. Wen YK (1976) Method for random vibration of hysteretic systems. Journal of the Engineering Mechanics Division 102(2):249-263

7. Baber TT, Wen YK (1981) Random vibration hysteretic, degrading systems. Journal of the Engineering Mechanics Division 107(6):1069-1087

8. Ismail M, Ikhouane F, Rodellar J (2009) The hysteresis bouc-wen model, a survey. Archives of Computational Methods in Engineering 16(2):161-188

9. Ikhouane F, Rodellar J (2007) Systems with hysteresis: analysis, identification and control using the Bouc-Wen model. John Wiley \& Sons

10. Cheng X, Hong W (2015) Research status of hysteretic nonlinear mode. Mechanical Engineer (2):184-188

11. Chassiakos AG, Masri SF, Smyth AW, Caughey TK (1998) On-line identification of hysteretic systems. Journal of Applied Mechanics 65(1):194-203

12. Yang G, Spencer Jr BF, Caelson JD, Sain MK (2002) Large-scale mr fluid dampers: modeling and dynamic performance considerations. Engineering Structures (24):309-323

13. Lu X, Zhou Q (2002) Dynamic analysis method of a combined energy dissipation system and its experimental verification. Earthquake Engineering \& Structural Dynamics 31(6):1251-1265

14. Zhu X, Lu X, Xu C (2011) Parametric identification of mild steel damper based on boucwen model. Structural Engineers 27(5):124-128

15. Rakotondrabe M (2011) Boucwen modeling and inverse multiplicative structure to compensate hysteresis nonlinearity in piezoelectric actuators. IEEE Transactions on Automation ence \& Engineering $8(2): 428-431$

16. Bagley RL, Torvik PJ (1983) Fractional calculus - a different approach to the analysis of viscoelastically damped structures. Aiaa Journal 21(5):741-748

17. Di Paola M, Pirrotta A, Valenza A (2011) Viscoelastic behavior through fractional calculus: an easier method for best fitting experimental results. Mechanics of materials 43(12):799-806

18. Sasso M, Palmieri G, Amodio D (2011) Application of fractional derivative models in linear viscoelastic problems. Mechanics of Time-Dependent Materials 15(4):367-387

19. Guo JWW, Daniel Y, Montgomery M, Christopoulos C (2016) Thermal-mechanical model for predicting the wind and seismic response of viscoelastic dampers. Journal of Engineering Mechanics 142(10):04016067

20. Lewandowski R, Łasecka-Plura M (2016) Design sensitivity analysis of structures with viscoelastic dampers. Computers \& Structures 164:95-107
21. Xu J, Li J (2016) Stochastic dynamic response and reliability assessment of controlled structures with fractional derivative model of viscoelastic dampers. Mechanical Systems and Signal Processing 72:865896

22. Kang S, Wu H, Li Y, Yu S, Yang X, Yao J (2020) A fractional-order normalized bouc-wen model for piezoelectric hysteresis nonlinearity. arXiv preprint arXiv:200304917

23. Hatchell BK, Mauss FJ, Amaya IA, Skorpik JR, Silvers KL, Marotta SA (2012) Missile captive carry monitoring and helicopter identification using a capacitive microelectromechanical systems accelerometer. Structural Health Monitoring $11(2): 213-224$

24. Zhang Y, Spanos PD (2020) Efficient response determination of a m-d-o-f gear model subject to combined periodic and stochastic excitations. International Journal of Non-Linear Mechanics 120:103378, DOI 10.1016/j.ijnonlinmec.2019. 103378

25. Yang J (2013) Vibration analysis on multi-mesh gear-trains under combined deterministic and random excitations. Mechanism and Machine Theory 59:20-33, DOI 10.1016/j.mechmachtheory.2012.08. 005

26. Xu Y, Liu Q, Guo G, Xu C, Liu D (2017) Dynamical responses of airfoil models with harmonic excitation under uncertain disturbance. Nonlinear Dynamics 89(3):1579-1590, DOI 10. 1007/s11071-017-3536-8

27. Harne RL, Dai Q (2017) Characterizing the robustness and susceptibility of steady-state dynamics in post-buckled structures to stochastic perturbations. Journal of Sound and Vibration 395:258271

28. Dai Q, Harne RL (2018) Investigation of direct current power delivery from nonlinear vibration energy harvesters under combined harmonic and stochastic excitations. Journal of intelligent material systems and structures 29(4):514-529

29. Caughey TK (1963) Equivalent linearization techniques. The Journal of the Acoustical Society of America 35(11):1706-1711

30. Kong F, Spanos PD (2021) Stochastic response of hysteresis system under combined periodic and stochastic excitation via the statistical linearization method. ASME Journal of Applied Mechanics Accepted, DOI doi:https://doi.org/10.1115/1. 4049836

31. Zhang Y, Spanos PD (2020) A linearization scheme for vibrations due to combined deterministic and stochastic loads. Probabilistic Engineering Mechan- 
ics 60:103028

32. Kong F, Spanos PD (2020) Response spectral density determination for nonlinear systems endowed with fractional derivatives and subject to colored noise. Probabilistic Engineering Mechanics 59:103023

33. Roberts JB, Spanos PD (2003) Random vibration and statistical linearization. Courier Corporation

34. Spanos P, Zeldin B (1997) Random vibration of systems with frequency-dependent parameters or fractional derivatives. Journal of Engineering Mechanics 123(3):290-292 
Figures

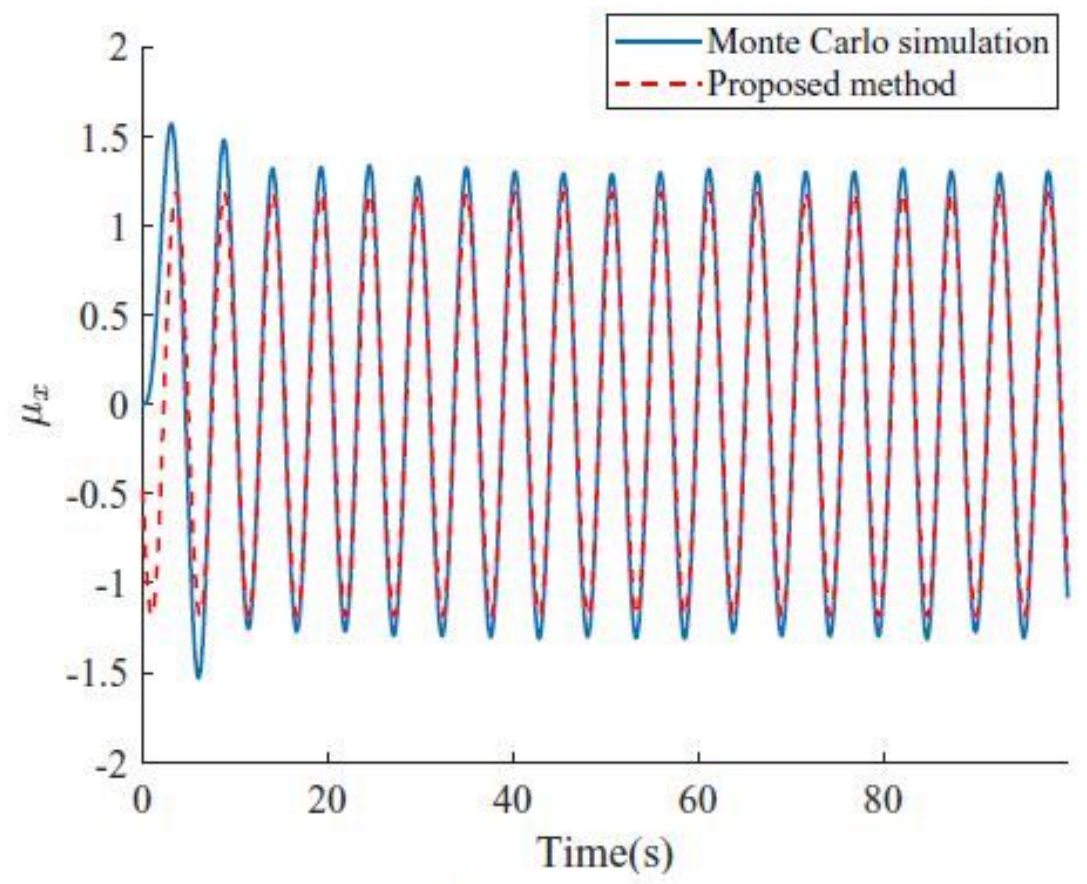

(a) Mean response $\mu_{x}$

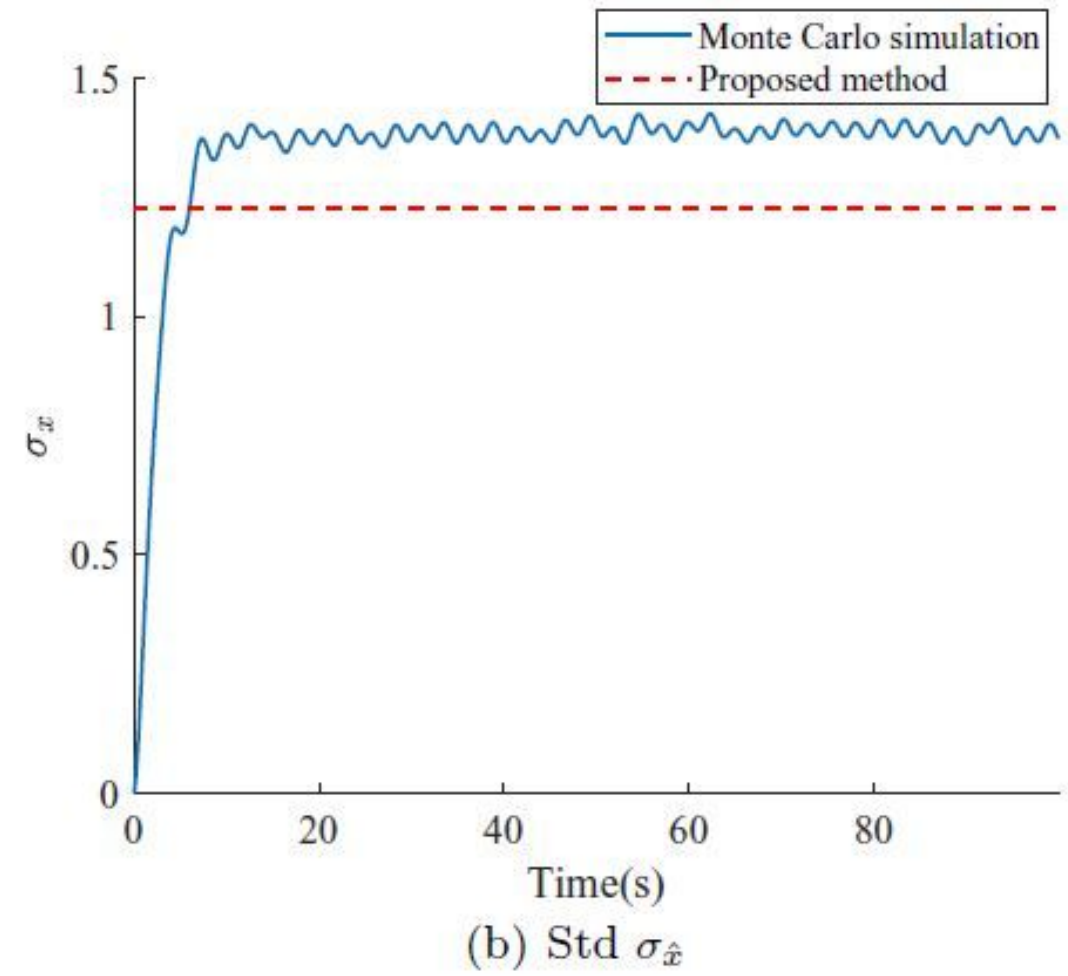

\section{Figure 1}

Displacement of the softening Bouc-Wen system sub- jected to combined excitation. 


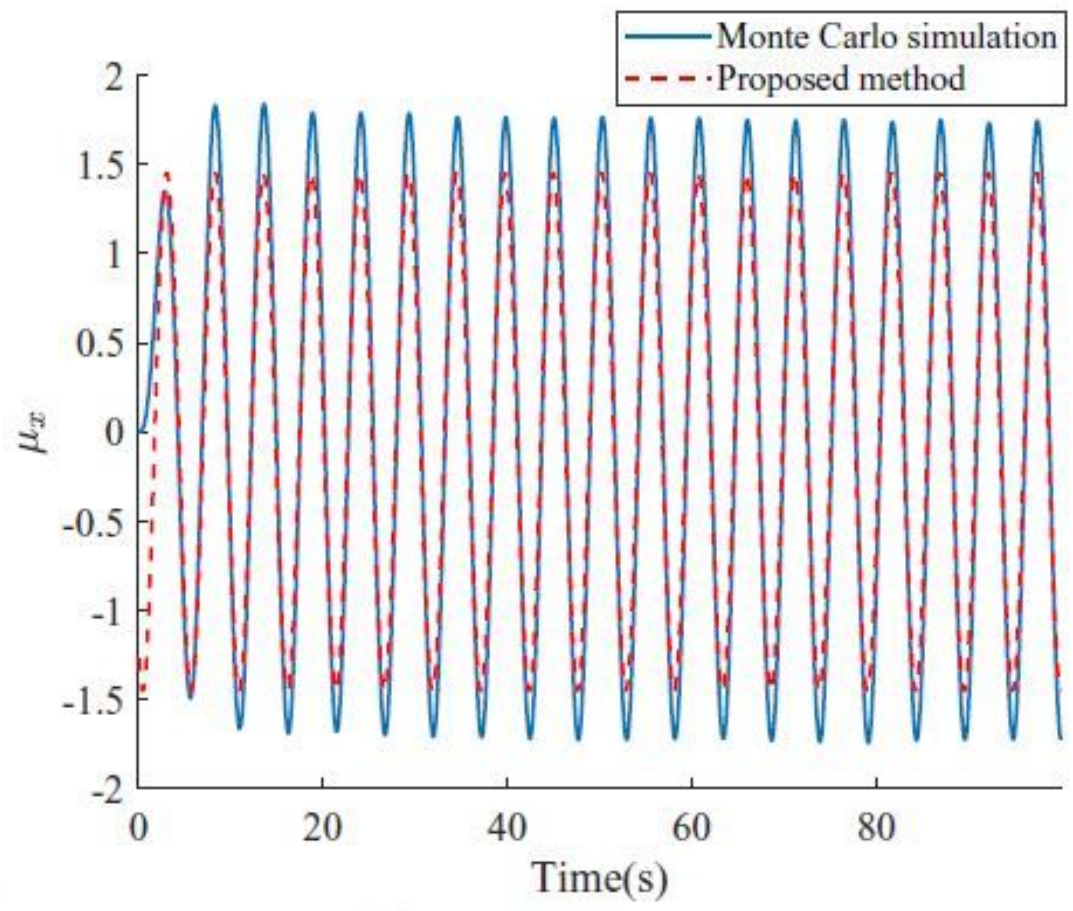

(a) Mean response $\mu_{x}$

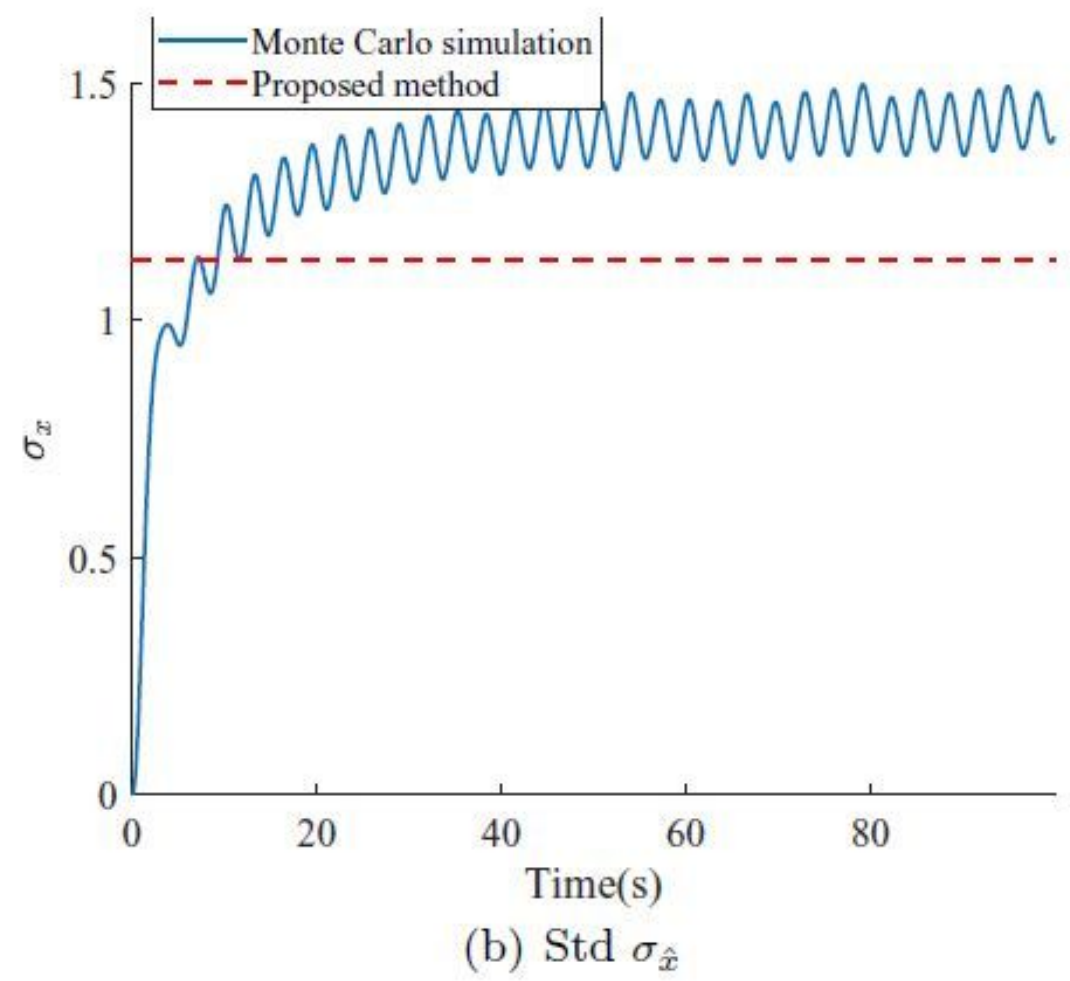

Figure 2

Displacement of the hardening Bouc-Wen system sub- jected to combined excitation. 


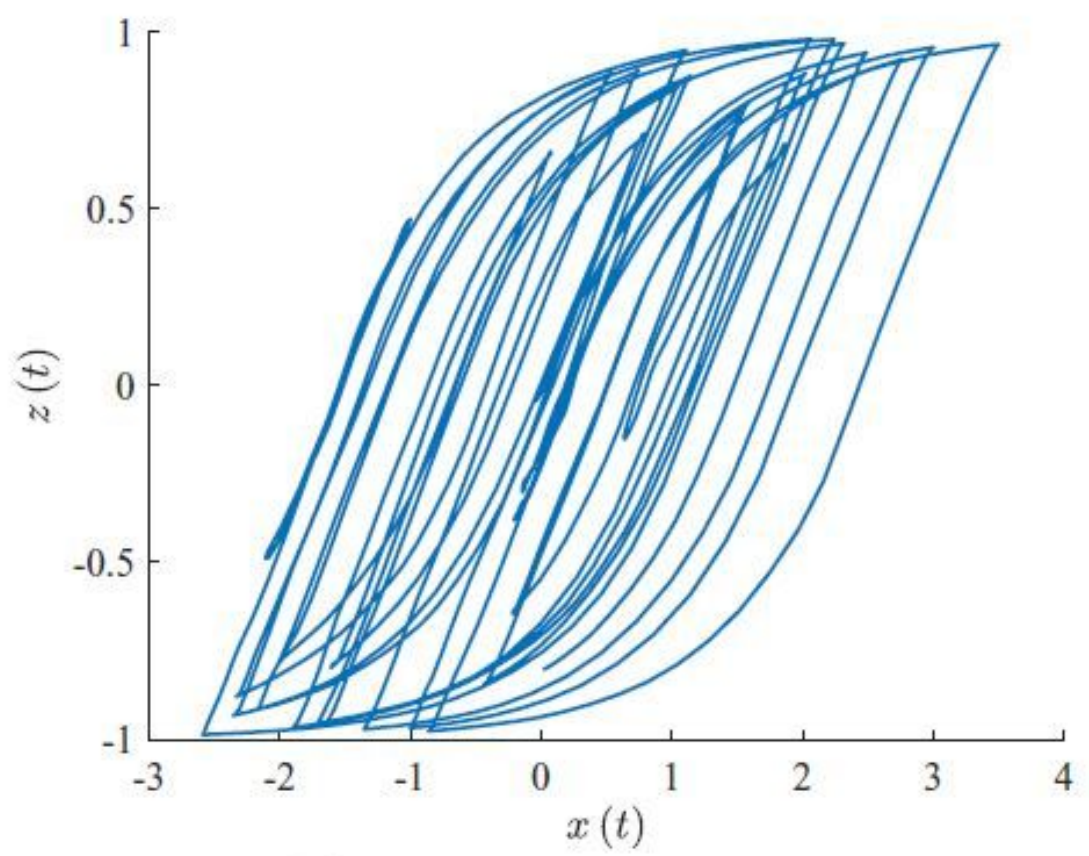

(a) Softening hysteretic loop

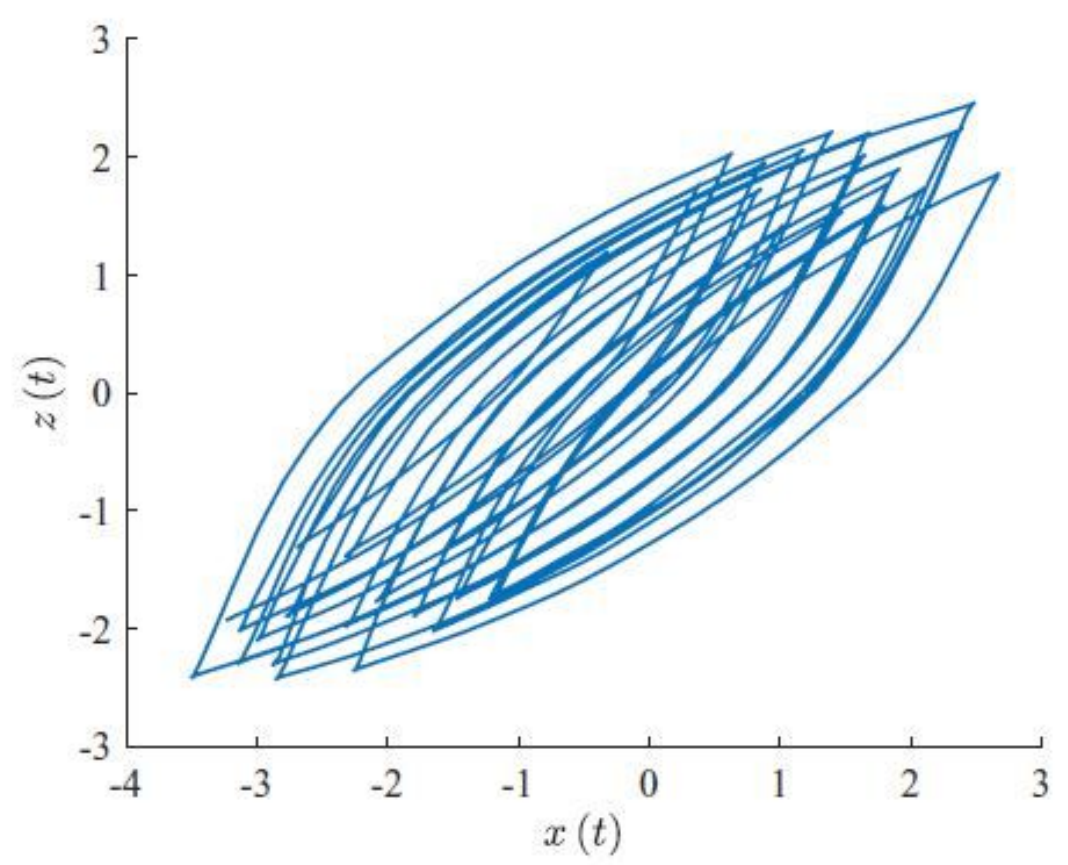

(b) Hardening hysteretic loop

\section{Figure 3}

Hysteretic loops of the Bouc-Wen dynamic system subjected to combined harmonic and stochastic excitation. 


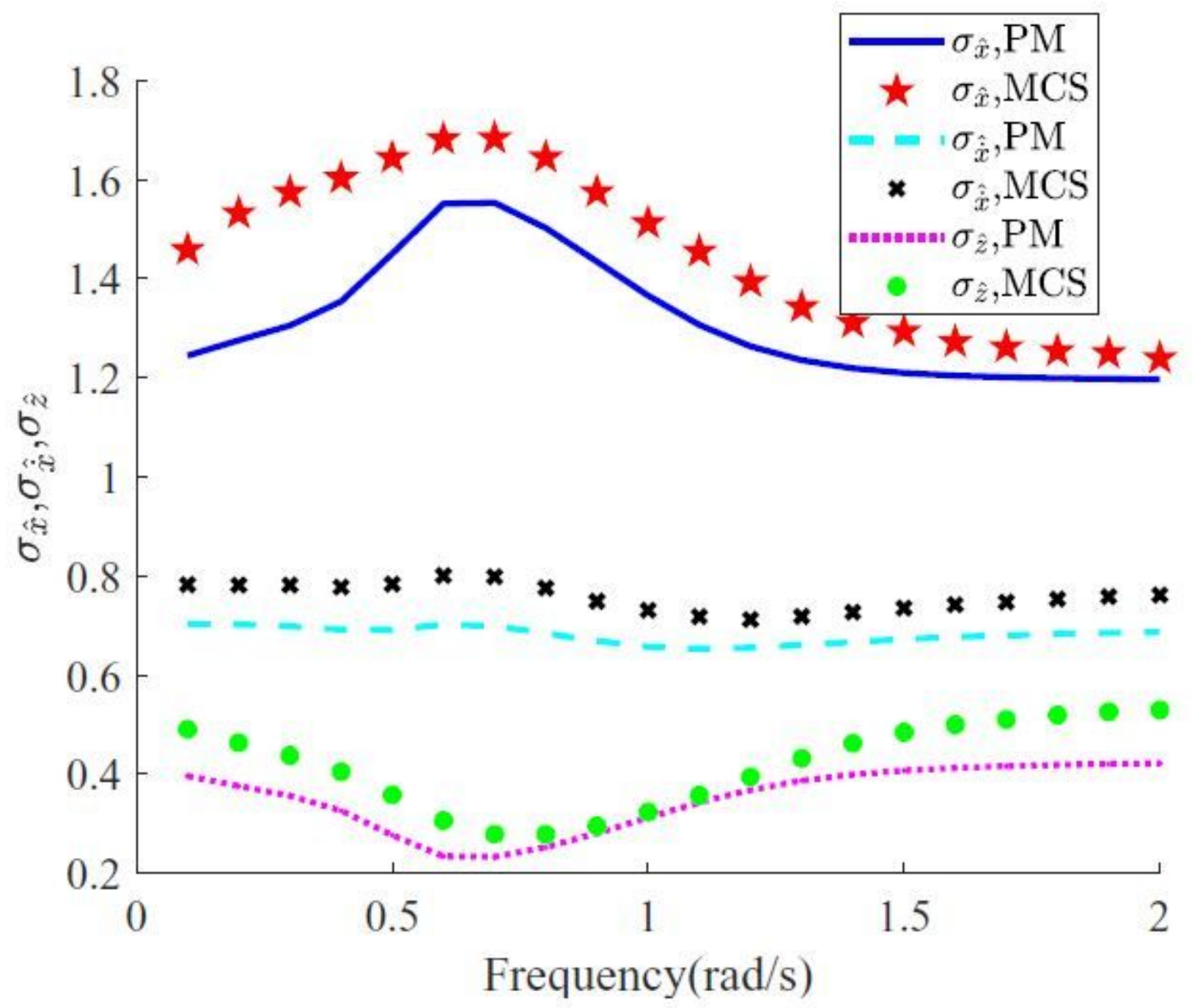

Figure 4

Standard deviation of the stochastic response compo- nent of a softening Bouc-Wen system subjected to combined stochastic excitation and harmonic excitation with di囚erent frequencies 


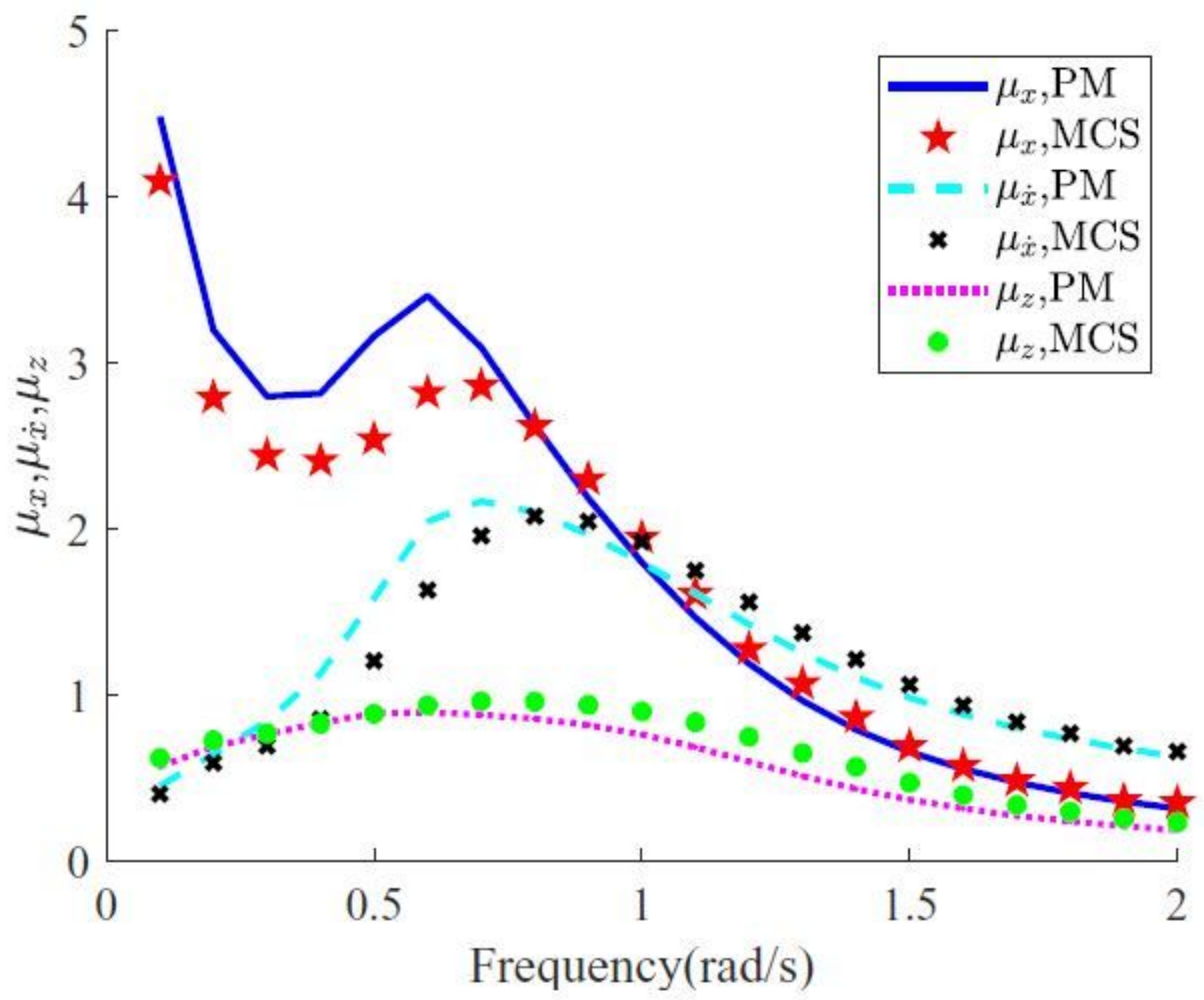

Figure 5

Amplitude of the deterministic response component of a softening Bouc-Wen system subjected to combined stochas- tic excitation and harmonic excitation with diðerent frequen- cies 


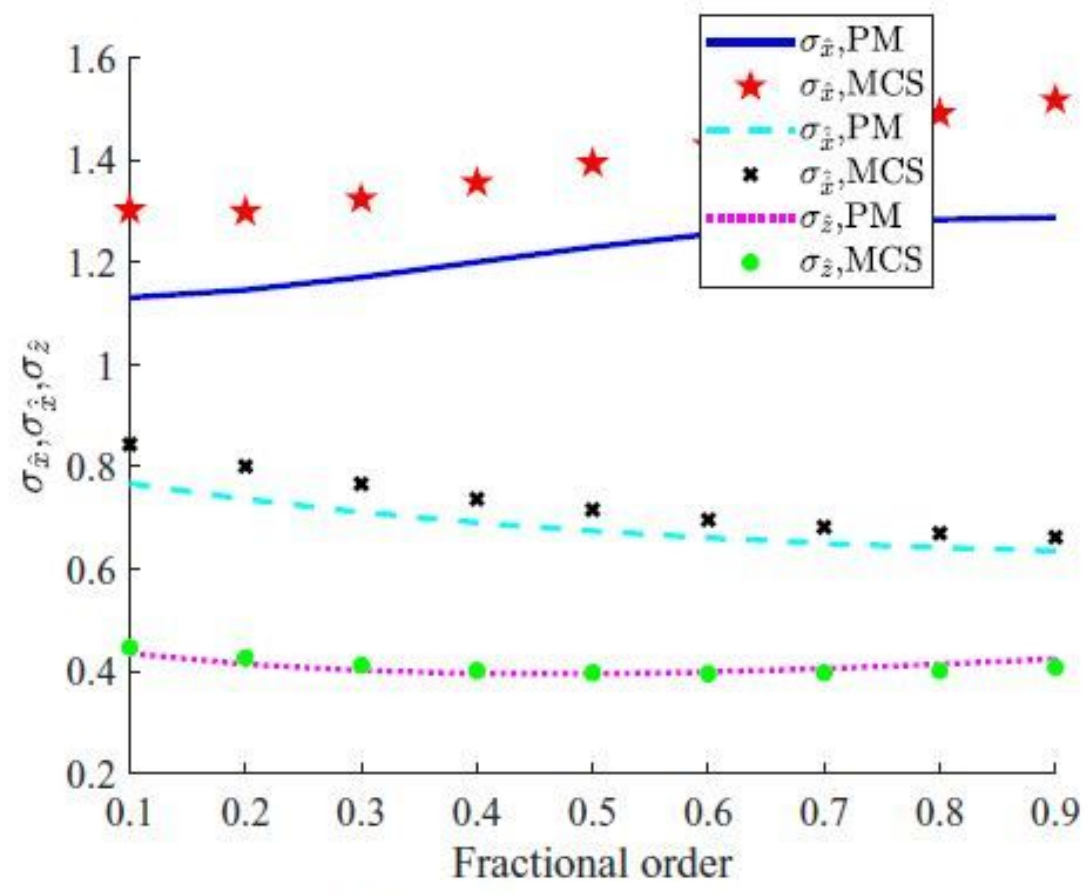

(a) softening system

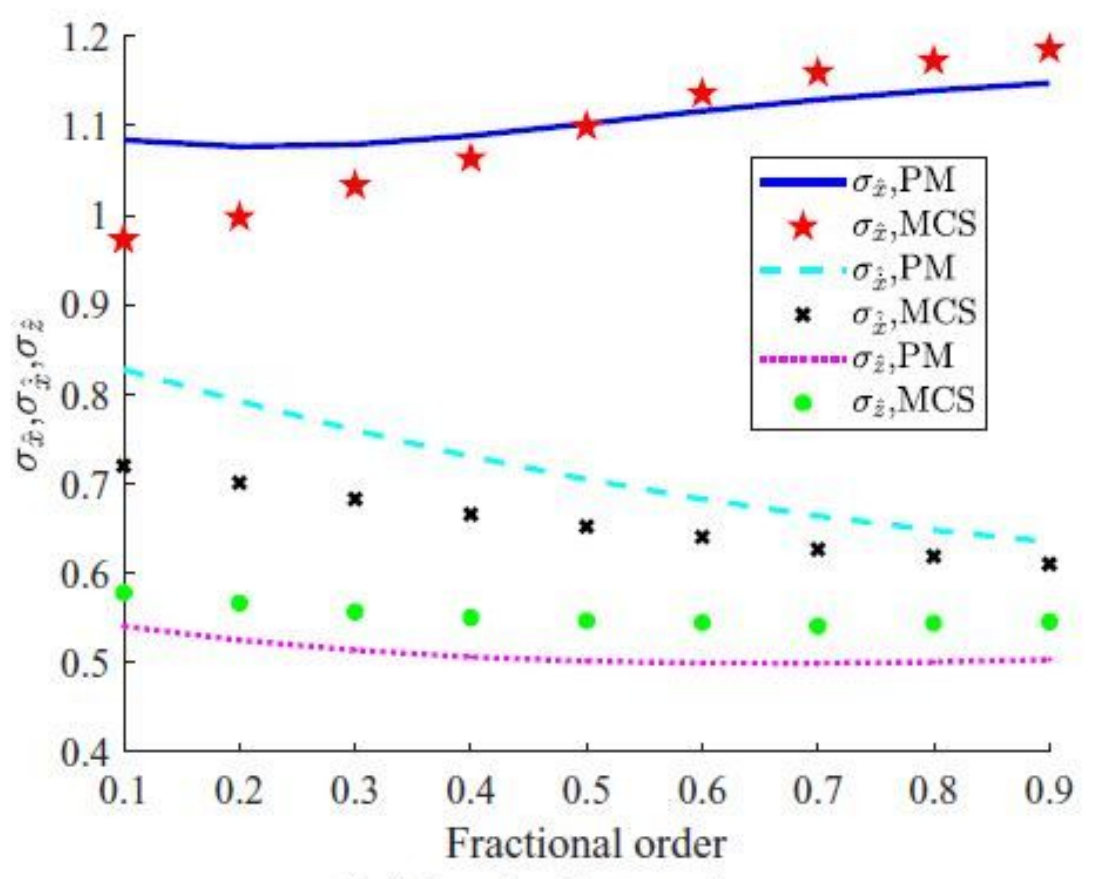

(b) hardening system

\section{Figure 6}

Standard deviation of the stochastic response compo- nent of softening and hardening Bouc-Wen systems subjected to combined stochastic excitation and harmonic excitation with di囚erent fractional orders 


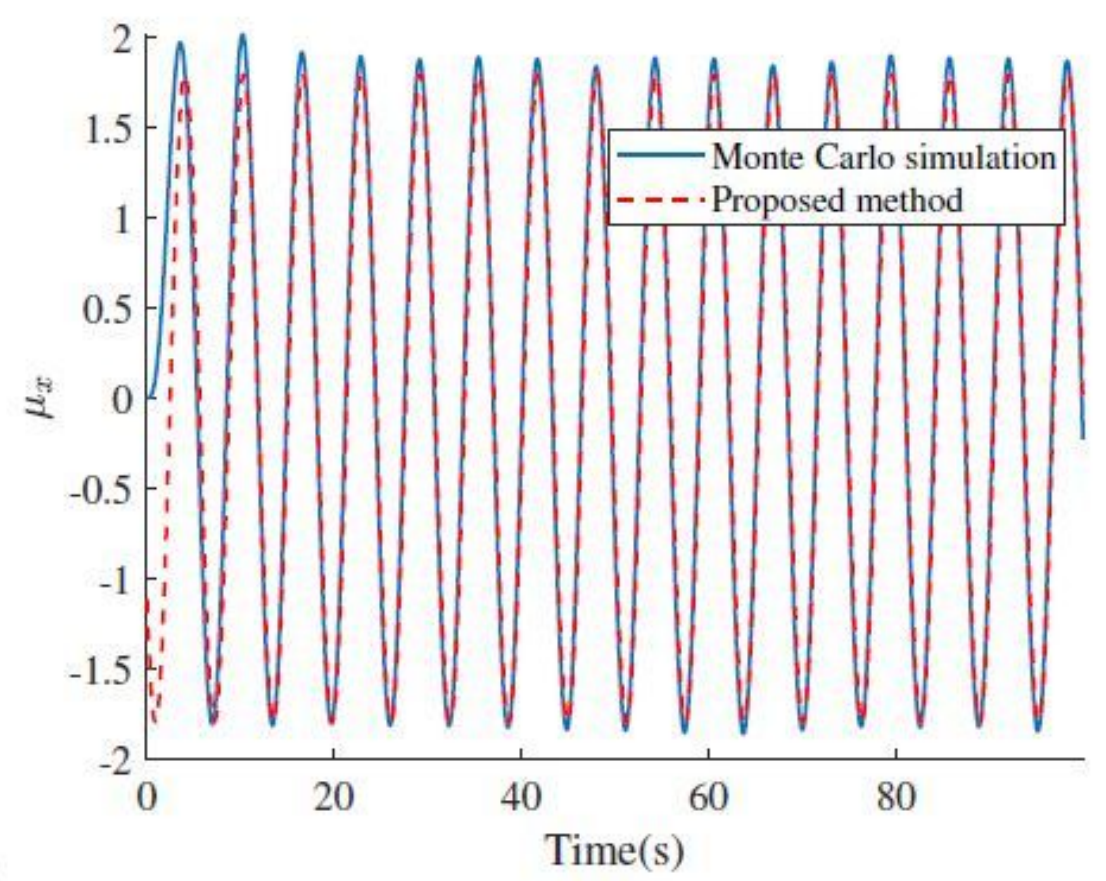

(a) Mean response $\mu_{x}$

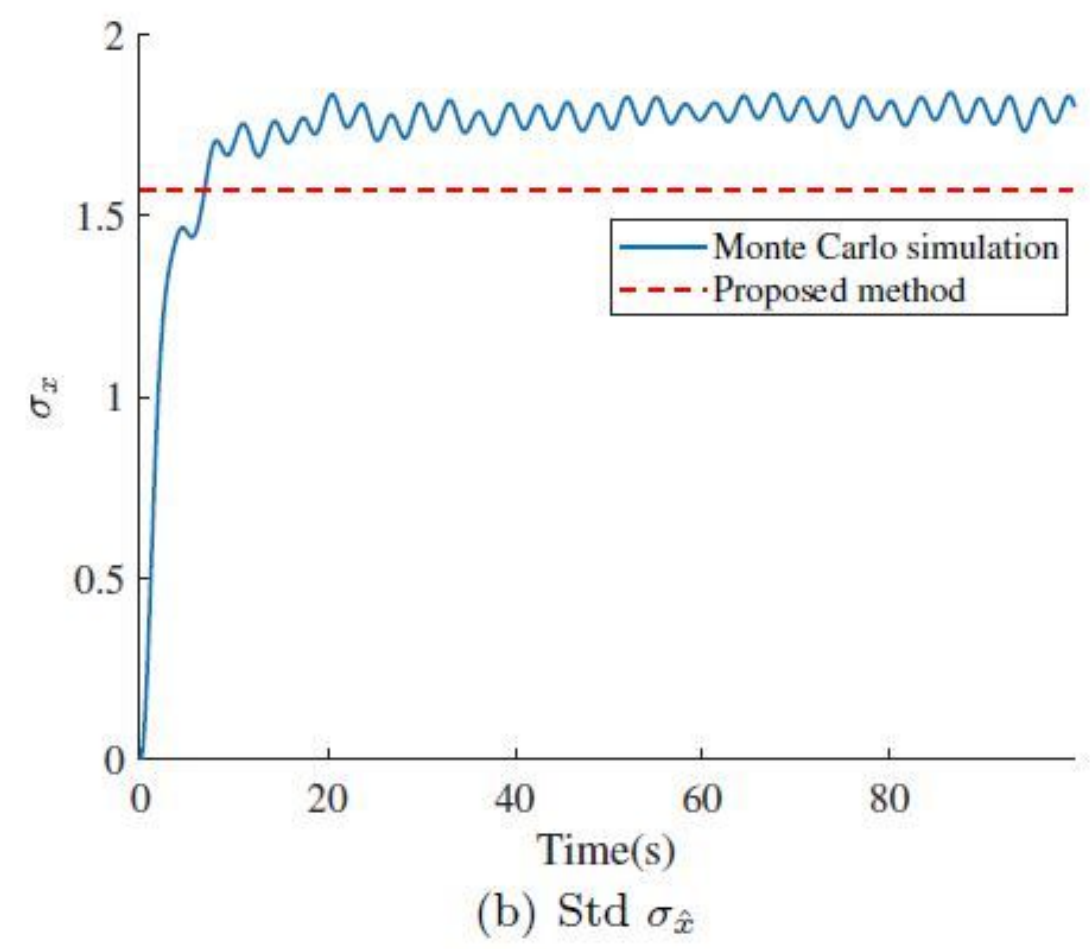

Figure 7

Displacement of the softening Bouc-Wen system sub- jected to combined excitation. 


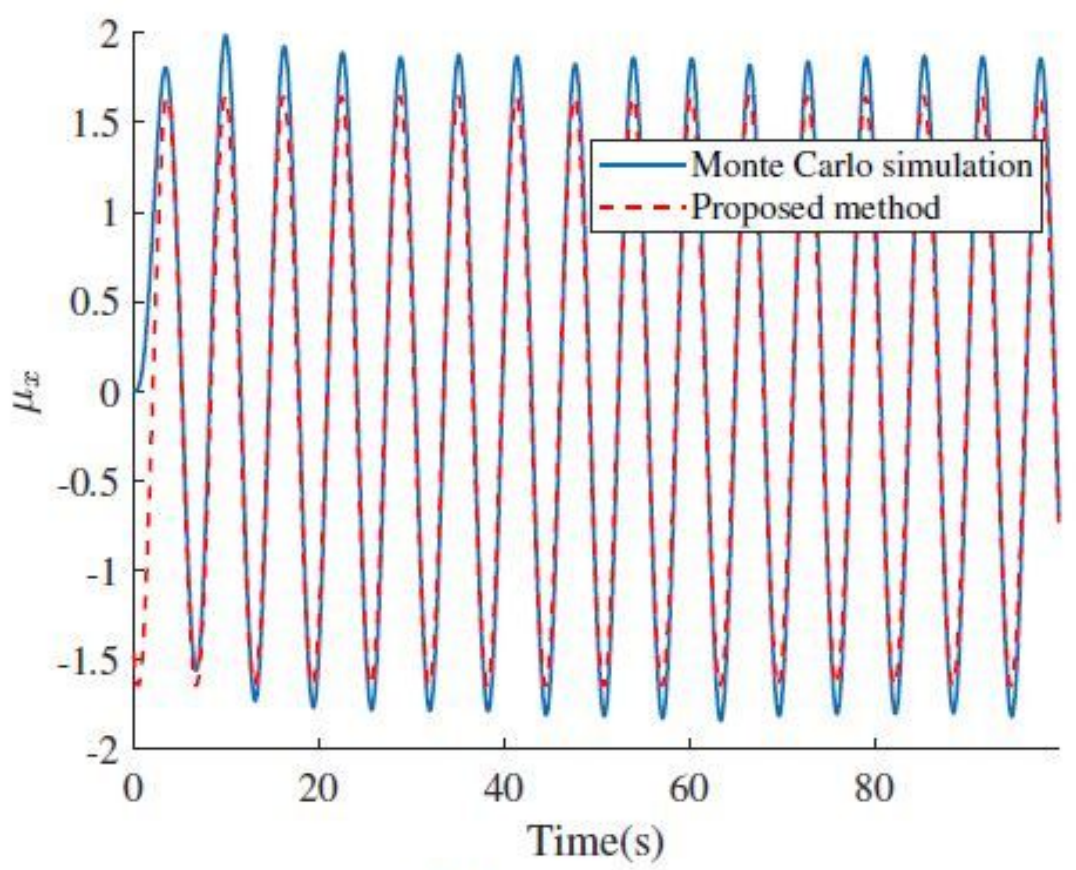

(a) Mean response $\mu_{x}$

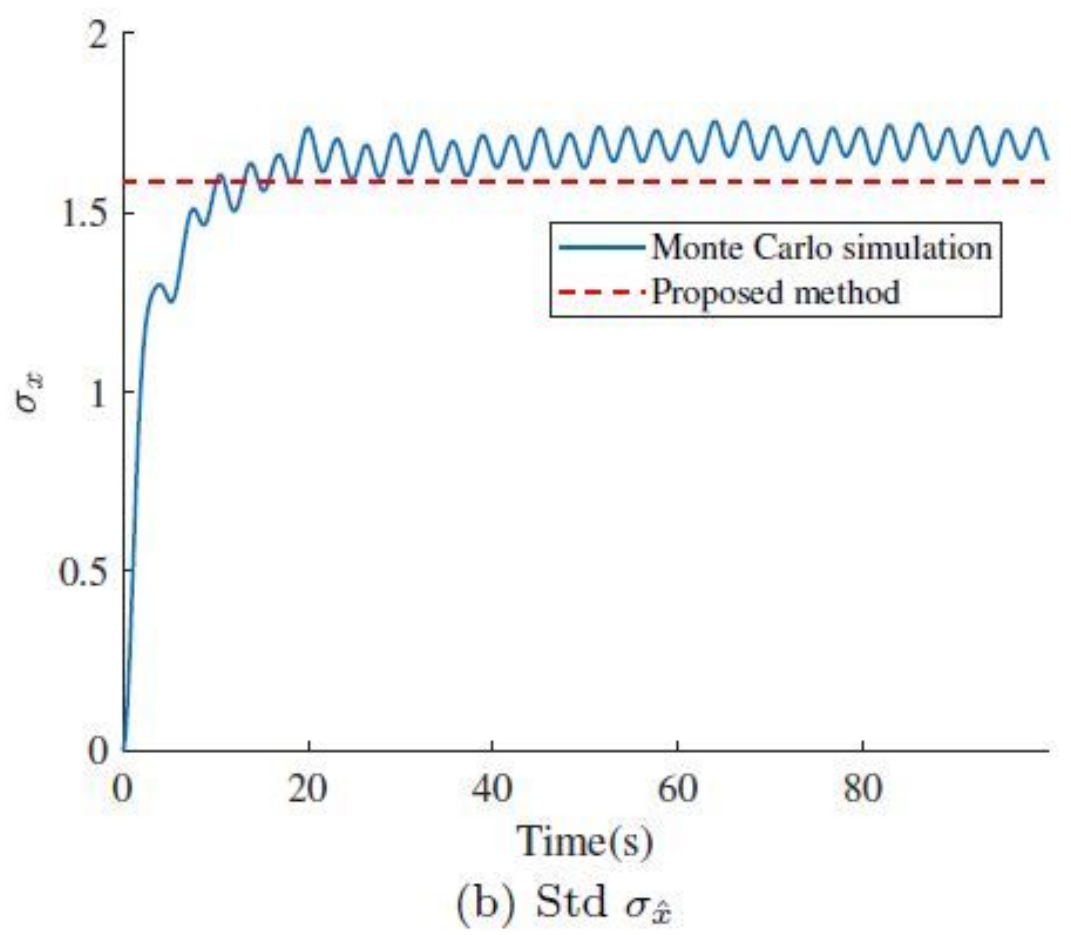

Figure 8

Displacement of the hardening Bouc-Wen system sub- jected to combined excitation. 


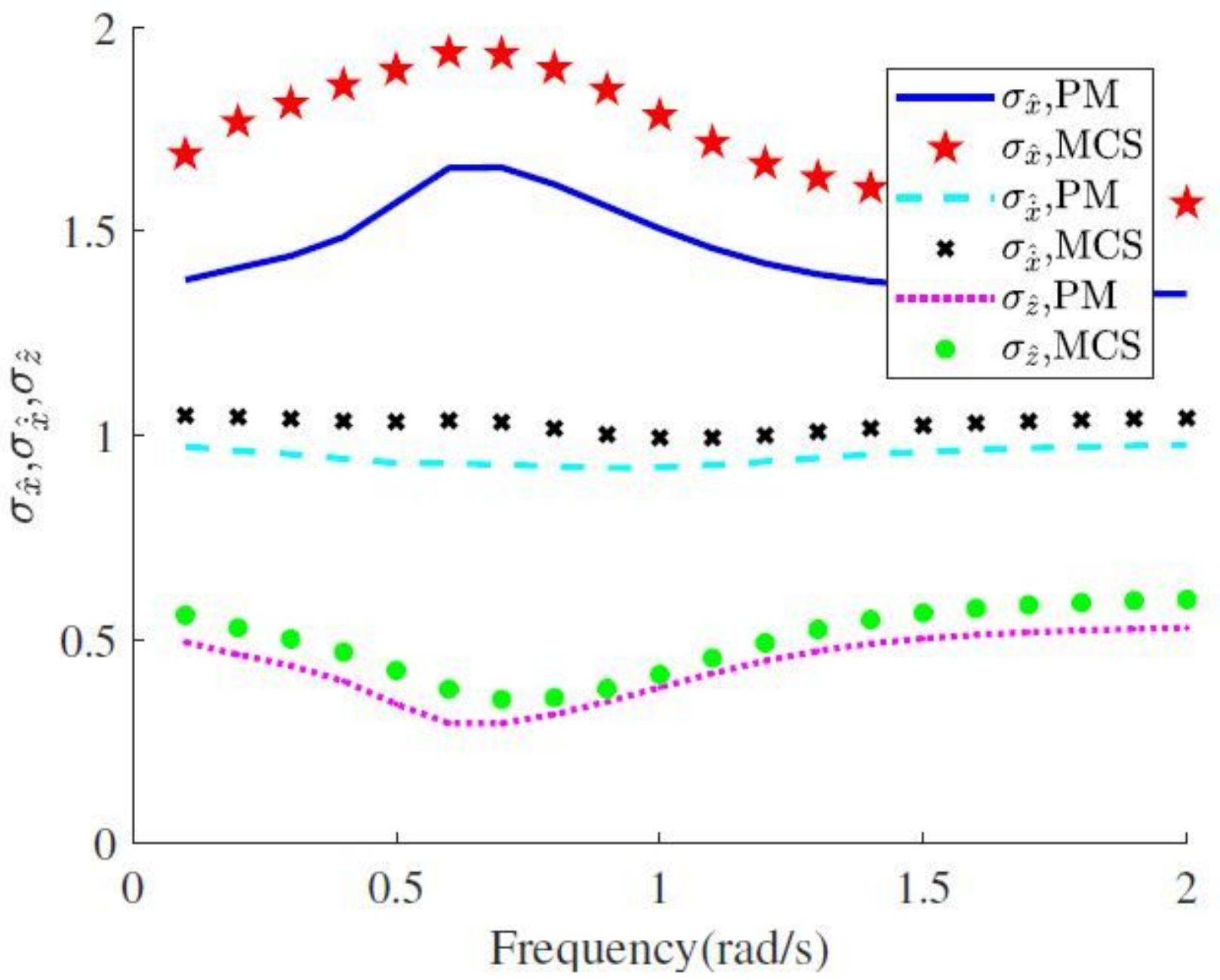

Figure 9

Standard deviation of the stochastic response compo- nent of a softening Bouc-Wen system subjected to combined stochastic excitation and harmonic excitation with di囚erent frequencies 


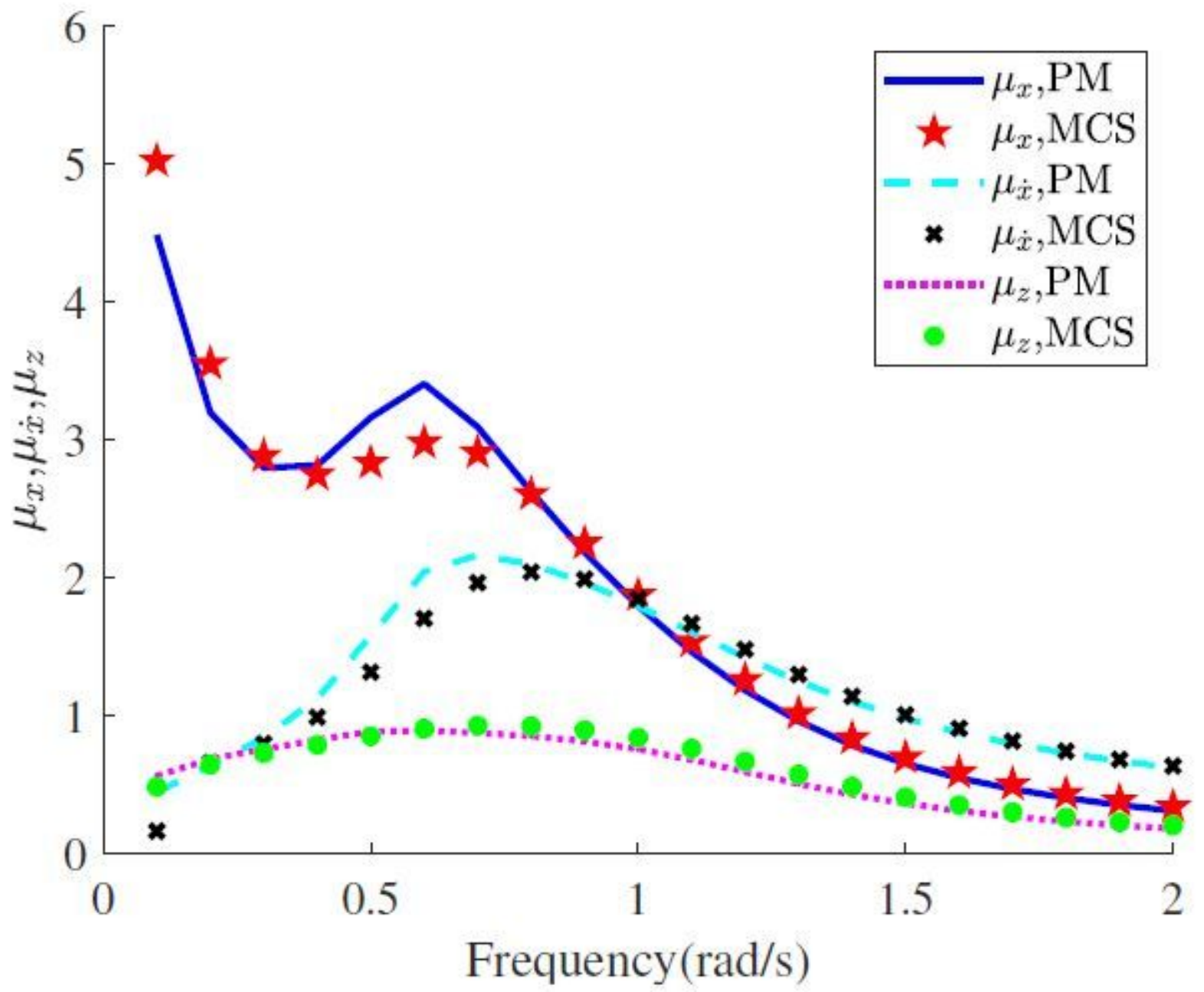

Figure 10

Amplitude of the deterministic response compo- nent of a softening Bouc-Wen system subjected to combined stochastic excitation and harmonic excitation with di囚erent frequencies 


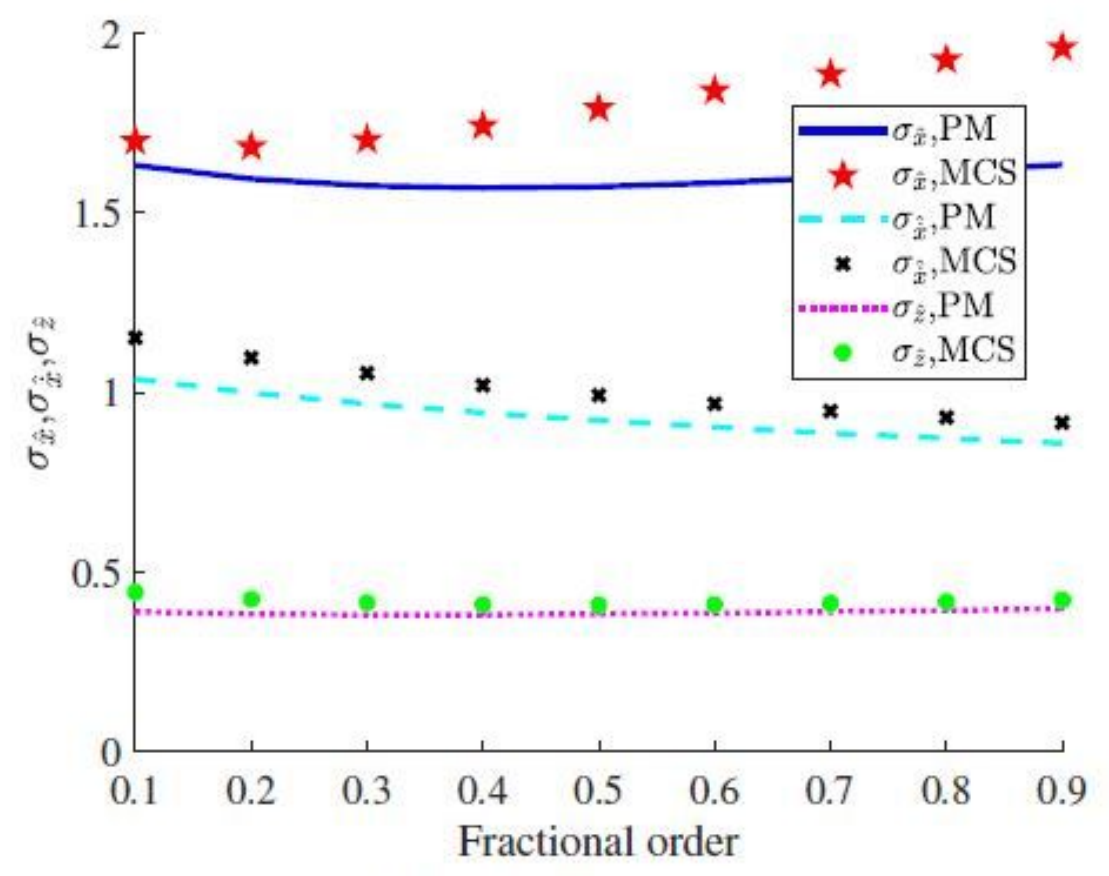

(a) softening system

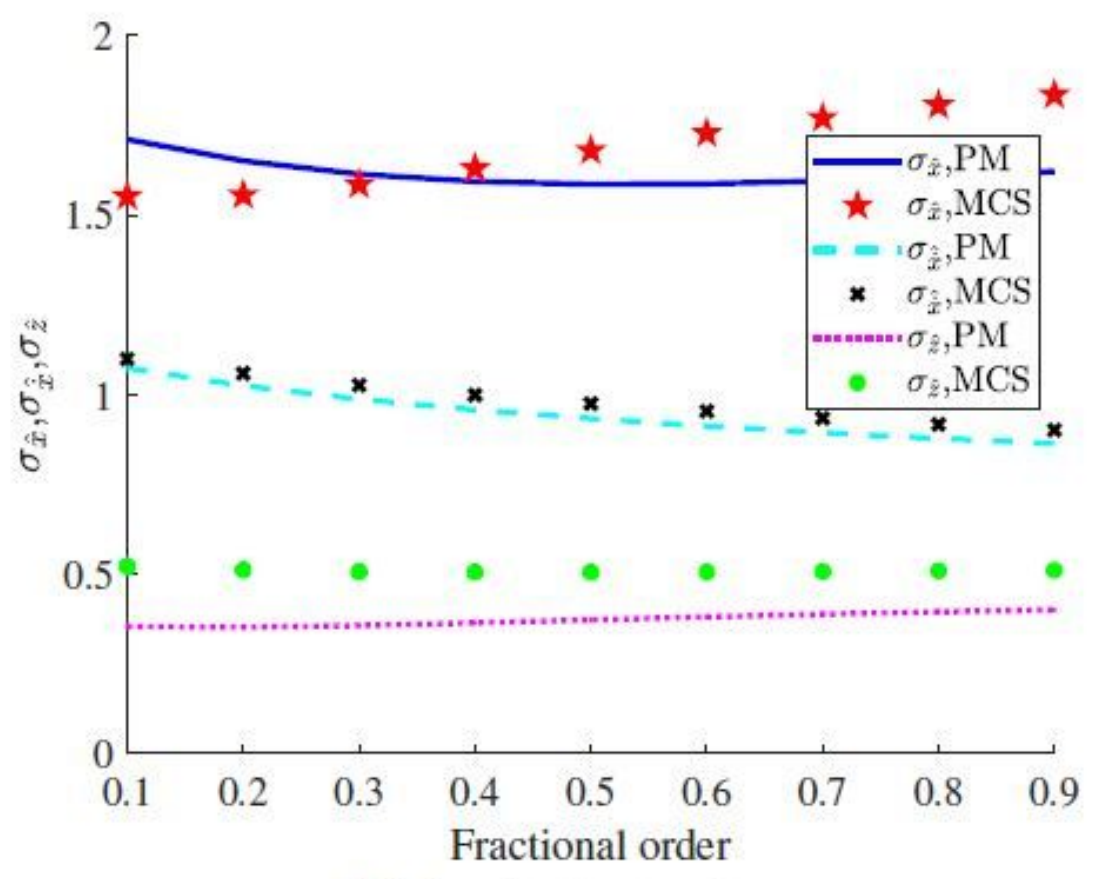

(b) hardening system

\section{Figure 11}

Standard deviation of the stochastic response compo- nent of softening and hardening Bouc-Wen systems subjected to combined stochastic excitation and harmonic excitation with di囚erent fractional orders 